\title{
Eine Verfassungsbeschwerde gegen den Bundesgerichtshof - Stehen Richterliche Unabhängigkeit und Gesetzesbindung der Richter in Deutschland zur Disposition der Exekutive?*
}

\section{Zusammenfassung}

Die richterliche Unabhängigkeit ist in Gefahr. Angesichts der Diskussion um den Rechtsstaatsmechanismus in der Europäischen Union gewinnt diese Gefahr makropolitische Bedeutung. Blickt man allerdings in die Details der europäischen Justizsysteme, ist diese Gefahr nicht neu. Sie wurde bisher nur entweder verkannt, oder verdrängt. Die im Folgenden abgedruckte Verfassungsbeschwerde von Thomas SchulteKellinghaus, Richter am Oberlandesgericht in Freiburg, rückt in das juristische Bewusstsein und Gewissen, wie groß die Gefahren für unabhängige richterliche Kontrolle in einem von Schlüsselindikatoren richterlicher Erledigung und der notorischen Überlastung von Justizsystemen geprägten Gerichtsalltag sind. Grundlage des Verfahrens: die Präsidentin eines Gerichts erteilt einem unabhängigen Richter eine Abmahnung und rügt ihn, nicht im Einklang mit den numerischen Erfordernissen justizieller Erledigungspraxis zu handeln. Nicht richterliche Sorgfalt, sondern systemische Funktionalität scheint vorherrschendes Paradigma. Angesichts der Rechtsprechung des Europäischen Gerichtshofs in Luxemburg aber in Sachen polnischer und ungarischer Justizreform, fragt sich, ob es künftig nicht näherliegt, ähnliche Fälle europäischer justizieller Kontrolle zu unterwerfen?

\section{Résumé}

L'indépendance du pouvoir judiciaire est en danger. Au vu de la discussion sur le mécanisme de l'État de droit dans l'Union européenne, ce danger prend une importance macro-politique. Cependant, si l'on examine les détails des systèmes judiciaires européens, ce danger n'est pas nouveau. Jusqu'à présent, elle n'a été que mal jugée ou supprimée. La plainte constitutionnelle de Thomas Schulte-Kellinghaus, juge au tribunal régional supérieur de Fribourg (Oberlandesgericht Freiburg), dont voici le texte, fait prendre conscience aux juristes des dangers que représente un contrôle judiciaire indépendant dans une vie judiciaire quotidienne caractérisée par des indicateurs clés de l'exécution des décisions de justice et par la surcharge notoire des systèmes judiciaires. La base de la procédure : la présidence d'un tribunal adresse un avertissement à un juge indépendant, le réprimandant pour ne pas avoir agi conformément aux exigences numériques de la décharge judiciaire. Ce n'est pas la diligence judiciaire mais la fonctionnalité systémique qui semble être le paradigme dominant. Toutefois, au vu de la jurisprudence de la Cour de justice des Communautés européennes à Luxembourg en matière de réforme judiciaire polonaise et hongroise, la ques-

* Richter am Oberlandesgericht. 
tion se pose de savoir s'il ne serait pas plus approprié à l'avenir de soumettre des affaires similaires au contrôle judiciaire européen?

\begin{abstract}
Judicial independence is in danger. In view of the discussion about the rule of law mechanism in the European Union, this danger gains macro-political significance. However, if one looks into the details of the European judicial systems, this danger is not new. It has only been either misjudged or suppressed so far. The constitutional complaint by Thomas Schulte-Kellinghaus, judge at the Higher Regional Court in Freiburg (Oberlandesgericht Freiburg), printed in the following, brings into the legal consciousness and conscience how great the dangers are for independent judicial control in an everyday judicial life characterised by key indicators of judicial execution and the notorious overloading of judicial systems. Basis of the procedure: the presidency of a court issues a warning to an independent judge, reprimanding him for not acting in accordance with the numerical requirements of judicial discharge. Not judicial diligence but systemic functionality seems to be the prevailing paradigm. However, in view of the case law of the European Court of Justice in Luxembourg (Poland and Hungary), the question arises whether it would not be more appropriate in future to subject similar cases to European judicial control?
\end{abstract}

Stefan Braum

\title{
I. Kurzbegründung - Ein Überblick
}

Der am 25.6.1954 geborene Beschwerdeführer ist seit 2002 als Richter in verschiedenen Zivilsenaten des Oberlandesgerichts Karlsruhe tätig. Seine richterliche Überzeugung führt seit Beginn seiner Tätigkeit am Oberlandesgericht dazu, dass er im Schnitt mehr Zeit für die Bearbeitung der ihm zugewiesenen Fälle benötigt als manche anderen Richterinnen und Richter. Diesen zeitlichen Mehraufwand kann der Beschwerdeführer nur zum Teil durch eigene Mehrarbeit ausgleichen.

Mit einem schriftlichen Bescheid vom 26.1.2012 (Anlage 5) hat die Präsidentin des Oberlandesgerichts Karlsruhe dem Beschwerdeführer vorgehalten, er habe bei den ihm als Berichterstatter oder Einzelrichter zugewiesenen Verfahren zu weniger Erledigungen beigetragen als der Durchschnitt der anderen Richterinnen und Richter am Oberlandesgericht Karlsruhe. In der Zeit vom 1.1.2008 bis zum 31.12.2011 habe er mit insgesamt 186 Erledigungen von Berufungsverfahren nur 63,7\% der durchschnittlichen Erledigungen $(291,9)$ am Oberlandesgericht Karlsruhe erzielt. Die Präsidentin hat den Beschwerdeführer ermahnt, in der Zukunft eine „Erledigungsleistung“ zu erzielen, die dem Durchschnitt der Kolleginnen und Kollegen entspreche. Inhalt und Begründung der Maßnahme hat die Präsidentin in einem von ihr selbst verfassten und unterzeichneten Schriftsatz vom 29.4.2013 (Anlage 7, S. 3) wie folgt konkretisiert: 


\section{„Durch die gesetzliche Vorgabe der Personalausstattung und das tatsächliche Fallaufkommen wird aber der - auch für den Berufungsführer - verbindliche Maßstab aufgestellt, wie viel der einzelne Richter in seiner jeweiligen Funktion insgesamt zu erledigen hat. "}

Die dienstrechtliche Maßnahme hatte das Ziel, den Beschwerdeführer zu einer Änderung seiner Rechtsanwendung in einer Vielzahl von Fällen zu veranlassen. Denn nur durch eine Änderung der Rechtsanwendung könnte er die ihm zugewiesenen Fälle mit weniger Zeit und entsprechend höheren Erledigungszahlen bearbeiten. Die Präsidentin wusste dies insbesondere aus einem ausführlichen Gespräch mit dem Beschwerdeführer vom 30.4.2010 (vgl. die Anlage 8). Eine Änderung der Rechtsanwendung würde der richterlichen Überzeugung des Beschwerdeführers widersprechen. Er würde seine wichtigste richterliche Pflicht, nämlich die Pflicht zur überzeugungsgemäßen Rechtsanwendung, die mit der Pflicht zur Gesetzesbindung identisch ist, verletzen. Der Beschwerdeführer ist daher nicht berechtigt, der Ermahnung der Präsidentin Folge zu leisten. Der heutige Präsident des Oberlandesgerichts Karlsruhe, Alexander Riedel, vertritt jedoch als Vertreter des Landes Baden-Württemberg in den anhängigen Gerichtsverfahren weiterhin die Auffassung, der Beschwerdeführer sei dienstrechtlich verpflichtet, der schriftlichen Ermahnung vom 26.1.2012 Folge zu leisten. Vorhalt und Ermahnung enthalten gleichzeitig eine konkludente Androhung disziplinarischer Maßnahmen für den Fall, dass sich der Beschwerdeführer der Ermahnung nicht beugt.

Der Beschwerdeführer hat im dienstgerichtlichen Verfahren gemäß $\S 26$ Abs. 3 DRiG beantragt, die Unzulässigkeit der Maßnahme vom 26.1.2012 festzustellen. Der Antrag des Beschwerdeführers ist vor den Dienstgerichten erfolglos geblieben. Die Urteile des Dienstgerichtshofs in Stuttgart vom 21.5.2019 und des Bundesgerichtshofs vom 12.5.2020 verletzen die richterliche Unabhängigkeit des Beschwerdeführers (Art. 97 Abs. 1 GG). Die verfassungsrechtliche Funktion der richterlichen Unabhängigkeit für die Realisierung der Gesetzesbindung des Richters (Art. 20 Abs. 3 GG) ist vom Dienstgerichtshof und vom Bundesgerichtshof verkannt worden. Die Gerichte haben sich zudem im dienstgerichtlichen Verfahren mit großen Teilen der Schriftsätze des Beschwerdeführers nicht beschäftigt, ohne dass Gründe für eine rechtliche Unerheblichkeit des Beschwerdeführer-Sachvortrags erkennbar wären. Wesentliche Teile des Sachverhalts, aus denen sich Inhalt und Ziel der Maßnahme der Präsidentin vom 26.1.2012 ergeben, sind vom Bundesgerichtshof, vom Dienstgerichtshof für Richter und vom Dienstgericht für Richter nicht berücksichtigt worden. Das gilt insbesondere für die von der Präsidentin geforderte angebliche ,gesetzliche Vorgabe der Personalausstattung“ (Anlage 7, S. 3, siehe das Zitat oben).

Der Beschwerdeführer hat bereits gegen ein früheres (zurückverweisendes) Urteil des Bundesgerichtshofs im dienstgerichtlichen Verfahren Verfassungsbeschwerde eingelegt. Diese frühere Verfassungsbeschwerde vom 30.1.2018 hat die erste Kammer des Zweiten Senats des Bundesverfassungsgerichts mit Beschluss vom 9.3.2018 - 2 BvR 174/18 - nicht zur Entscheidung angenommen, weil der Rechtsweg noch nicht erschöpft sei. Die vorliegende Verfassungsbeschwerde richtet sich gegen die neuen Urteile des Bundesgerichtshofs und des Dienstgerichtshofs, die nach der Zurückverwei- 
sung und nach der Entscheidung des Bundesverfassungsgerichts vom 9.3.2018 ergangen sind.

\section{Sachverhalt}

Der Beschwerdeführer war in der Vergangenheit am Oberlandesgericht Karlsruhe in verschiedenen Zivilsenaten tätig, vom 29.7.2002 bis zum 30.6.2007 im 15. Zivilsenat in Karlsruhe, vom 1.7.2007 bis zum 31.3.2011 im 4. Zivilsenat an der Außenstelle in Freiburg, und seit dem 1.4.2011 (bis heute) im 9. Zivilsenat ebenfalls in Freiburg.

Am 30.4.2010 fand ein ausführliches Gespräch zwischen der Präsidentin des Oberlandesgerichts Karlsruhe, Prof. Dr. Christine Hügel, dem Beschwerdeführer und seinem damaligen Senatsvorsitzenden Christoph Ertl statt. In diesem Gespräch ging es um die Arbeitsbelastung des Beschwerdeführers, um seine richterlichen Überzeugungen und um deren Auswirkungen auf seinen Zeitbedarf pro Fall (vgl. den Gesprächsvermerk Anlage 8).

Am 18.10.2011 übergab die Präsidentin gelegentlich eines aus anderen Gründen vereinbarten Gesprächs einen auf den 12.10.2011 datierten Vermerk (Anlage 9). Der Vermerk enthielt verschiedene Vorwürfe, die angebliche Fehler oder Versäumnisse des Beschwerdeführers in seiner richterlichen Tätigkeit betrafen. Von der in diesem Vermerk erwähnten Sonderprüfung hatte der Beschwerdeführer vor dem Gespräch vom 12.10.2011 keine Kenntnis. Nach dem Gespräch bat der Beschwerdeführer - vor einer beabsichtigten Stellungnahme zu dem Vermerk - um verschiedene ergänzende Informationen, die er trotz mehrerer Anfragen nur unvollständig erhielt.

Am 26.1.2012 erließ die Präsidentin - ohne vorherige Stellungnahme des Beschwerdeführers - den als „Vorhalt und Ermahnung“ bezeichneten Bescheid vom 26.1.2012 (Anlage 5). Die dem Antragsteller für die Zeit von 2008 bis 2011 zugeordneten Erledigungszahlen seien unzureichend; er sei verpflichtet, ein am „Durchschnittspensum“ aller Richterinnen und Richter des Oberlandesgerichts Karlsruhe gemessenes „Erledigungspensum" zu erreichen.

Zwischen dem Beschwerdeführer und der Justizverwaltung des Landes Baden-Württemberg war immer - auch in den gerichtlichen Verfahren - außer Streit, dass der Beschwerdeführer einen hohen, deutlich über 41 Stunden pro Woche liegenden, persönlichen Arbeitseinsatz leistet. Wegen teilweise unzulänglicher Berichterstattungen hat der Präsident des Oberlandesgerichts Karlsruhe, Alexander Riedel, dies in einem Schreiben vom 2.2.2017 an die Badischen Neuesten Nachrichten (Anlage 10) in Karlsruhe noch einmal klargestellt. Der hohe persönliche Arbeitseinsatz führt in einem Nebeneffekt u. a. dazu, dass vom Beschwerdeführer stammende Urteile und Beschlüsse in sehr großer Zahl in Fachzeitschriften veröffentlicht werden. Eine Auswertung der veröffentlichten Gerichtsentscheidungen in Juris zeigt beispielsweise für den Zeitraum von 2012 bis 2017, dass vom 9. Zivilsenat mehr Entscheidungen veröffentlicht wurden, als von jedem anderen Zivilsenat des Oberlandesgerichts Karlsruhe, 
wobei mehr als 90 Prozent der veröffentlichten Entscheidungen des 9. Zivilsenats vom Beschwerdeführer entworfen oder verfasst wurden.

Der Beschwerdeführer hat gegen den Bescheid vom 26.1.2012 am 24.2.2012 Widerspruch eingelegt, den er mit Schriftsatz seines Rechtsanwalts vom 13.4.2012 begründet hat. Die Präsidentin wies den Widerspruch am 20.4.2012 zurück (Anlage 6). Der Beschwerdeführer erhob hiergegen sodann Klage zum Dienstgericht für Richter am Landgericht Karlsruhe mit dem Antrag, die Unzulässigkeit des Bescheids vom 26.1.2012 und des Widerspruchsbescheids vom 20.4.2012 wegen Verletzung der richterlichen Unabhängigkeit festzustellen. Das Dienstgericht für Richter bei dem Landgericht Karlsruhe hat die Klage mit Urteil vom 4.12.2012 - RDG 6/12 - (Anlage 4) abgewiesen.

Gegen das Urteil des Dienstgerichts hat der Beschwerdeführer Berufung zum Dienstgerichtshof für Richter bei dem Oberlandesgericht Stuttgart eingelegt. Die Berufung ist mit Schriftsatz vom 11.3.2013 (Anlage 11) begründet worden. In einem Grundsatzschriftsatz vom 27.11.2013 (Anlage 12) hat der Beschwerdeführer konkretisiert, was eine Dienstaufsicht nach Zahlen für seine Rechtsanwendung bedeuten würde. Außerdem hat der Beschwerdeführer in diesem Schriftsatz in rechtlicher Hinsicht die Funktion der richterlichen Unabhängigkeit für die Realisierung der Gesetzesbindung erläutert. Im Schriftsatz vom 23.1.2015 (Anlage 13) sind die Anträge für das Berufungsverfahren konkretisiert worden.

Im Termin zur mündlichen Verhandlung vom 17.4.2015 (vgl. das Protokoll Anlage 14) hat der Beschwerdeführer mehrere Beweisanträge gestellt, die vom Dienstgerichtshof zurückgewiesen worden sind (vgl. die Anlage 14). Der Dienstgerichtshof hat die Berufung mit Urteil vom 17.4.2015 - DGH 2/13 - (Anlage 15) zurückgewiesen.

Gegen das Urteil des Dienstgerichtshofs hat der Beschwerdeführer Revision zum Bundesgerichtshof eingelegt. Die Revision ist mit Schriftsatz vom 23.9.2015 (Anlage 16) begründet worden. Die Gegenseite hat mit Schriftsatz vom 26.1.2016 (Anlage 17) erwidert. Im Termin zur mündlichen Verhandlung vom 7.9.2017 hat der Beschwerdeführer zur Rechtslage in einem Plädoyer Stellung genommen. Ein schriftliches Exemplar ist dem Senat in der Verhandlung übergeben worden (Anlage 18).

Mit Urteil vom 7.9.2017 hat der Bundesgerichtshof auf die Revision des Beschwerdeführers das Urteil des Dienstgerichtshofs aufgehoben und das Verfahren an den Dienstgerichtshof zurückverwiesen. Der Dienstgerichtshof habe eine ausreichende Aufklärung versäumt, wie die Präsidentin des Oberlandesgerichts die ihrem Bescheid zu Grunde liegenden Durchschnittszahlen ermittelt habe. Das schriftliche Urteil (Anlage 19) ist dem Beschwerdeführer am 2.11.2017 zugestellt worden. Der Beschwerdeführer hat gegen dieses Urteil am 13.11.2017 eine Anhörungsrüge (Anlage 20) erhoben. Diese ist vom Bundesgerichtshof mit Beschluss vom 13.12.2017 (Anlage 21) zurückgewiesen worden. Der Beschluss ist der Verfahrensbevollmächtigten des Beschwerdeführers am 2.1.2018 zugestellt worden (Anlage 22). 
Gegen das Urteil des Bundesgerichtshofs vom 7.9.2017 und gegen das Urteil des Dienstgerichtshofs vom 17.4.2015 hat der Beschwerdeführer am 30.1.2018 eine frühere Verfassungsbeschwerde erhoben (Anlage 23). Diese hat die erste Kammer des zweiten Senats mit Beschluss vom 9.3.2018 - 2 BvR 174/18 - nicht zur Entscheidung angenommen, weil der Rechtsweg vor den Dienstgerichten nach der Zurückverweisung durch den Bundesgerichtshof noch nicht erschöpft sei (Anlage 24). In dem beim Dienstgerichtshof in Stuttgart fortgesetzten Verfahren hat der Beschwerdeführer zur Berufungsbegründung mit Schriftsatz vom 16.4.2018 (Anlage 25) ergänzend vorgetragen. Mit einem weiteren Schriftsatz vom 9.5.2019 hat der Beschwerdeführer vorsorglich bereits früher gestellte Beweisanträge wiederholt (Anlage 26). Im Urteil vom 21.5.2019 (Anlage 3) hat der Dienstgerichtshof die Berufung des Beschwerdeführers erneut zurückgewiesen.

Gegen das Urteil des Dienstgerichtshofs hat der Beschwerdeführer erneut Revision zum Bundesgerichtshof eingelegt. Die Revision ist mit Schriftsatz vom 7.10.2019 begründet worden (Anlage 27). Im Termin zur mündlichen Verhandlung vom 12.5.2020 vor dem Bundesgerichtshof hat der Beschwerdeführer in einem Plädoyer zur Rechtslage Stellung genommen (vgl. die schriftliche Fassung Anlage 28). Im Urteil vom 12.5.2020 (Anlage 2) hat der Bundesgerichtshof die Revision des Beschwerdeführers zurückgewiesen. Das schriftliche Urteil ist dem Beschwerdeführer am 28.5.2020 zugestellt worden. Der Beschwerdeführer hat gegen dieses Urteil am 10.6.2020 eine Anhörungsrüge erhoben (Anlage 29). Die Anhörungsrüge hat der Bundesgerichtshof mit Beschluss vom 7.7.2020 als unbegründet zurückgewiesen (Anlage 30). Der Beschluss ist der Verfahrensbevollmächtigten des Beschwerdeführers am 20.7.2020 zugestellt worden (Anlage 31).

Zur Information des Bundesverfassungsgerichts:

Der Beschwerdeführer hat zwei weitere dienstgerichtliche Verfahren durchgeführt. Eine Klage hat sich gegen den Vermerk vom 12.10.2011 (Anlage 9) gerichtet, eine weitere Klage gegen die Durchführung der Sonderprüfung, die dem Vermerk vorausgegangen ist. Die in diesen Verfahren ergangenen Entscheidungen sind nicht Gegenstand der Verfassungsbeschwerde.

Im Verfahren des Dienstgerichts für Richter bei dem Landgericht Karlsruhe RDG 5/12 hat der Beschwerdeführer einen Teilerfolg erzielt. Im Urteil vom 4.12.2012 (Anlage 32) hat das Dienstgericht festgestellt, dass der Vermerk der Präsidentin vom 12.10.2011 und dessen Übergabe am 18.10.2011 sowie deren Widerspruchsbescheid vom 6.3.2012 insoweit unzulässig sind, als dem Beschwerdeführer vorgeworfen wird, die ihm zugeschriebenen Verfahren trotz erkennbarer oder mitgeteilter Eilbedürftigkeit nicht bearbeitet zu haben. Das Urteil ist rechtskräftig. In den Urteilsgründen hat das Dienstgericht festgestellt, dass aus den von der Kammer geprüften Unterlagen kein vom Beschwerdeführer unbearbeitetes eilbedürftiges Verfahren ersichtlich gewesen sei, und dass die Präsidentin weder vorgerichtlich noch im dienstgerichtlichen Verfahren ein möglicherweise eilbedürftiges, unbearbeitetes Verfahren genannt habe (Anlage 32, S. 18).Im Übrigen sind die Klagen des Beschwerdeführers in den beiden Parallelverfahren erfolglos geblieben (BGH RiZ (R) 1/15 und RiZ (R) 3/15). 


\section{Die Grundrechtsrügen}

Der Beschwerdeführer rügt die Verletzung seiner Grundrechte durch das Urteil des Bundesgerichtshofs vom 12.5.2020, durch das Urteil des Dienstgerichtshofs vom 21.5.2019 und durch das Urteil des Dienstgerichts für Richter vom 4.12.2012. Die Grundrechtsverstöße wurden durch die Entscheidung des Bundesgerichtshofs vom 7.7.2020 über die Anhörungsrüge des Beschwerdeführers nicht beseitigt.

- Die Entscheidungen verletzen die richterliche Unabhängigkeit des Beschwerdeführers (Art. 97 Abs. 1 GG). In der Rechtsprechung des Bundesverfassungsgerichts ist anerkannt, dass der Beschwerdeführer als betroffener Richter im Hinblick auf Art. 33 Abs. 5 GG berechtigt ist, eine Verletzung seiner Unabhängigkeit durch ein Organ der Exekutive im Wege einer Verfassungsbeschwerde geltend zu machen (vgl. BVerfG, Beschluss vom 29.2.1996 - 2 BvR 136/96 -, Rn. 12, zitiert nach Juris). Zum Schutzbereich von Art. 33 Abs. 5 GG gehört die richterliche Unabhängigkeit. Soweit im Folgenden verkürzend von ,,Verletzung der richterlichen Unabhängigkeit" gesprochen wird, ist die Verletzung von Art. 33 Abs. 5 GG i. V. m. Art. 97 Abs. 1 GG gemeint.

- Der Bundesgerichtshof und der Dienstgerichtshof haben das Grundrecht auf rechtliches Gehör des Beschwerdeführers (Art. 103 Abs. 1 GG) verletzt. Die Entscheidungen beruhen darauf, dass wesentliches Vorbringen des Beschwerdeführers nicht zur Kenntnis genommen und nicht berücksichtigt wurde.

- Die Entscheidungen sind willkürlich und verletzen das Grundrecht des Beschwerdeführers aus Art. 3 Abs. 1 GG. Der Umgang der Richter mit dem Sachverhalt und die darauf beruhende Subsumtion in den angegriffenen Entscheidungen sind unter keinem Gesichtspunkt rechtlich vertretbar; es drängt sich der Schluss auf, dass die Entscheidungen auf sachfremden Erwägungen beruhen.

- Die Entscheidungen missachten die Verpflichtung des Beschwerdeführers zur Gesetzesbindung (Art. 20 Abs. 3 GG), die ihm verbietet, sich dem Ansinnen der Präsidentin im Bescheid vom 26.1.2012 zu fügen. Aus der Gesetzesbindung ergibt sich zwar unmittelbar kein mit der Verfassungsbeschwerde rügefähiges Grundrecht des Beschwerdeführers; im Rahmen einer gemäß $§ 90$ BVerfGG zulässigen Verfassungsbeschwerde hat das Bundesverfassungsgericht jedoch die Kompetenz, zu prüfen, inwieweit ein Verstoß gegen eine Verfassungsnorm vorliegt, die nicht in $\S 90$ Abs. 1 BVerfGG genannt ist (vgl. Zuck, Das Recht der Verfassungsbeschwerde, 5. Aufl. 2017, Rn. 817 unter Hinweis auf die einschlägige Rechtsprechung des Bundesverfassungsgerichts; BVerfG, Beschluss vom 25.2.1964 - 2 BvR 411/61 -, Rn. 11, zitiert nach Juris). Vorliegend kommt der Gesetzesbindung des Beschwerdeführers eine besondere Bedeutung zu. Die Bedeutung der richterlichen Unabhängigkeit - und die Bedeutung der Verletzung der richterlichen Unabhängigkeit durch die Maßnahme der Präsidentin und durch die angegriffenen Gerichtsentscheidungen - erschließt sich erst im Zusammenhang mit dem Prinzip der Gesetzesbindung. 


\section{Die Zulässigkeit der Verfassungsbeschwerde}

Die Verfassungsbeschwerde ist zulässig.

1. Der Beschwerdeführer macht eine Verletzung der oben genannten Grundrechte durch die angegriffenen Entscheidungen geltend (§90 Abs. 1 BVerfGG). Dass eine Verletzung vorliegt, wird unten näher ausgeführt.

Ein Rechtsschutzinteresse ist gegeben. Der Beschwerdeführer ist weiterhin als Richter am Oberlandesgericht tätig, nach derzeitigem Stand voraussichtlich bis zum 30.6.2022 (Vollendung des 68. Lebensjahres). Eine der Verfassung entsprechende dienstgerichtliche Entscheidung ist notwendig, um die Grundlagen der richterlichen Tätigkeit des Beschwerdeführers für die Gegenwart und für die $\mathrm{Zu}$ kunft zu klären. Der Präsident des Oberlandesgerichts, Alexander Riedel, vertritt bis heute die Auffassung, der Beschwerdeführer sei verpflichtet, der Ermahnung vom 26.1.2012 Folge zu leisten. Würde der Bescheid vom 26.1.2012 unbeanstandet bestehen bleiben, würde die damit verbundene konkludente Androhung disziplinarischer Maßnahmen bei einer Fortsetzung der richterlichen Tätigkeit des Beschwerdeführers weiter Bestand haben.

2. Die Einlegungsfrist von einem Monat ( $\$ 93$ Abs. 1 BVerfGG) ist gewahrt. Zur Erschöpfung des Rechtswegs war die Erhebung einer Anhörungsrüge gegen die Entscheidung des Bundesgerichtshofs erforderlich. Denn der Verstoß des Bundesgerichtshofs gegen das Grundrecht des Beschwerdeführers auf rechtliches Gehör (Art. 103 Abs. 1 GG) ist ein wesentlicher Teil dieser Verfassungsbeschwerde (siehe unten VI.). Der Bundesgerichtshof hat die zulässige Anhörungsrüge (Anlage 29) mit Beschluss vom 7.7.2020 (Anlage 30) zurückgewiesen. Der Beschluss ist dem Beschwerdeführer am 20.7.2020 zugestellt worden (Anlage 31), so dass die Frist zur Einlegung der Verfassungsbeschwerde am 20.8.2020 abläuft. Diese Frist ist für die Zulässigkeit der Verfassungsbeschwerde auch insoweit maßgeblich, als - neben der Verletzung des rechtlichen Gehörs - gleichzeitig die Verletzung anderer Grundrechte gerügt wird (vgl. BVerfG, NJW 2005, 3059 - Queen Mary II).

3. Der Rechtsweg ist erschöpft ( $\$ 90$ Abs. 2 Satz 1 BVerfGG).

Der Beschwerdeführer hat die mögliche Gehörsrüge gegen die angegriffene Entscheidung des Bundesgerichtshofs erfolglos erhoben; ein anderes Rechtsmittel gegen das Urteil des Bundesgerichtshofs gibt es nicht.

4. Die Verfassungsbeschwerde ist außerdem zulässig gemäß $§ 90$ Abs. 2 Satz 2 1. Hs. BVerfGG; denn sie ist von allgemeiner Bedeutung im Sinne dieser Vorschrift. Die allgemeine Bedeutung ergibt sich daraus, dass die Entscheidung des Bundesverfassungsgerichts für die Rechtspraxis an deutschen Gerichten große Bedeutung haben wird (vgl. zur „allgemeinen Bedeutung“ die Beispiele aus der Rechtsprechungspraxis des Bundesverfassungsgerichts bei Zuck, Das Recht der Verfassungsbeschwerde, 5. Aufl. 2017, Rn. 774). 
a) Ungeachtet der eindeutigen Rechts- und Verfassungssituation (siehe unten) ist in den letzten Jahren bei vielen Richterinnen und Richtern in Deutschland eine Unsicherheit entstanden, ob und inwieweit sie ihre richterliche Überzeugung vom Gesetz und von der Anwendung des Gesetzes noch in den Vordergrund gegenüber einem Fall-erledigungsinteresse - stellen dürfen, wenn der Zeitbedarf für die Durchsetzung der richterlichen Überzeugung bei der Rechtsanwendung zu Arbeitsrückständen bei den zugewiesenen Verfahren führt oder führen könnte (vgl. dazu die Ausführungen im Plädoyer des Beschwerdeführers im ersten BGH-Termin vom 7.9.2017, Anlage 18). Die Entscheidung des Bundesverfassungsgerichts ist erforderlich, um diese Unsicherheit in der Richterschaft in Deutschland zu beseitigen. Diese Unsicherheit wird auch durch die Feststellungen, die das Bundesverfassungsgericht in der Deal-Entscheidung vom 19.3.2013 zur Rechtspraxis von Strafrichtern getroffen hat, dokumentiert; neuere rechtstatsächliche Untersuchungen zeigen, dass sich die damals vom Bundesverfassungsgericht beanstandete Praxis inzwischen allenfalls teilweise geändert hat.

b) Der Beschwerdeführer führt seit als mehr acht Jahren ein Verfahren gegen das Land Baden-Württemberg. Es ist an der Zeit, dass eine Entscheidung des Bundesverfassungsgerichts Klarheit an deutschen Gerichten schafft. Ein anderes Verfahren beim Bundesverfassungsgericht, das zu einer verfassungsrechtlichen Klärung der gegenständlichen Fragen führen würde, ist nicht ersichtlich.

c) Die Präsidentinnen und Präsidenten der Oberlandesgerichte haben sich in Besprechungen mit dem Fall beschäftigt. Die Reaktion der Mehrheit der OLGPräsidenten auf eine Umfrage der Neuen Richtervereinigung im Jahr 2017 zeigt, dass die Präsidentinnen und Präsidenten sich die Möglichkeit einer Dienstaufsicht nach Zahlen erhalten wollen, bei der Zweckmäßigkeitsgesichtspunkte im Vordergrund stehen sollen (vgl. die Anlagen 33, 34). Eine baldige Entscheidung des Bundesverfassungsgerichts ist notwendig für die Praxis der Dienstaufsicht durch die Präsidentinnen und Präsidenten der Oberlandesgerichte.

d) Es geht um die Praxis der Gesetzesbindung der Richter (vgl. insbesondere den Grundsatzschriftsatz des Beschwerdeführers im Verfahren vor dem Dienstgerichtshof, Anlage 12, dort Ziff. 2 bis Ziff. 7, S. 3 - S. 12). Es ist von allgemeiner Bedeutung, dass Richterinnen und Richter ihrer Pflicht zur Gesetzesbindung generell auch dann folgen dürfen (und müssen), wenn Bearbeitungsrückstände entstehen. Und es ist von allgemeiner Bedeutung, dass Richterinnen und Richter ihrer Pflicht zur Gesetzesbindung generell auch dann noch folgen dürfen (und müssen), wenn die eigene Überzeugung deutlich mehr Zeit kostet, als die Überzeugung von Kolleginnen und Kollegen, die keine „Zahlenprobleme“ haben. Die Erkenntnis, dass ein Richter, der für überzeugungsgemäße Entscheidungen Zeit benötigt, bei „Rückständen“ nicht für die Funktionstüchtigkeit der Justiz verantwortlich ist (so für die Strafjustiz ausdrücklich Landau, 
NStZ 2015, 665, 670), ist unter Richterinnen und Richtern und erst recht in der Justizverwaltung in großem Umfang verloren gegangen.

\section{Begründetheit - Verletzung der richterlichen Unabhängigkeit (Art. 97 Abs. 1 GG)}

\section{Richterliche Unabhängigkeit und Gesetzesbindung - Der verfassungsrechtli- che Maßstab für die Entscheidung des Bundesgerichtshofs}

a) Die angegriffene Entscheidung des Bundesgerichtshofs ist am verfassungsrechtlichen Grundsatz der sachlichen Unabhängigkeit des Richters (Art. 97 Abs. 1 GG) zu messen. Der Inhalt dieses Verfassungsprinzips ist in der Rechtsprechung des Bundesverfassungsgerichts geklärt. Der Exekutive ist jede vermeidbare Einflussnahme auf die Rechtsanwendung des Richters untersagt (BVerfG, Beschluss vom 4.6.1969 - 2 BvR 33/66, 2 BvR 387/66 -, Rn. 44, zitiert nach Juris; BVerfG, Beschluss vom 17.1.2013 - 2 BvR 2576/11 -, Rn. 8, zitiert nach Juris). Eine Einflussnahme kann nicht nur durch direkte Weisung, sondern auch mittelbar, indirekt und subtil erfolgen. Da auch eine solche indirekte Einflussnahme Auswirkungen auf die Rechtsanwendung des Richters und auf seine Entscheidung haben kann, ist der Exekutive auch jede mittelbare, subtile und psychologische Einflussnahme verboten (BVerfG, Beschluss vom 22.6.2006 - 2 BvR 957/05 -, Rn. 7, zitiert nach Juris; BVerfG, Beschluss vom 17.1.2013 - 2 BvR 2576/11 -, Rn. 8, zitiert nach Juris). Art. 97 Abs. 1 GG gibt dem Richter daher u. a. auch ein Abwehrrecht gegen eine überfordernde Einflussnahme bei der Zuweisung eines „Arbeitspensums“ (BVerfG, Beschluss vom 23.5.2012 - 2 BvR 610/12, 2 BvR 625/12 -, zitiert nach Juris).

b) Das Verbot einer Einflussnahme auf die Rechtsanwendung des Richters kennt keine Schranke. Insbesondere ergibt sich aus den Befugnissen der Exekutive zur Dienstaufsicht gemäß $§ 26$ Abs. 1 und Abs. 2 DRiG weder eine Schranke der richterlichen Unabhängigkeit noch eine Konkretisierung des verfassungsrechtlichen Begriffs der richterlichen Unabhängigkeit. Vielmehr wird auf der einfachrechtlichen Ebene durch $\S 26$ Abs. 1 2. Hs. DRiG der verfassungsrechtliche Vorrang der richterlichen Unabhängigkeit gegenüber den Befugnissen der Dienstaufsicht klargestellt (vgl. BVerfG, Beschluss vom 22.10.1974 - 2 BvR 147/70 -, Rn. 47, zitiert nach Juris; anders die Formulierung des Bundesgerichtshofs in der angegriffenen Entscheidung vom 12.5.2020, Anlage 2, Rn. 24). Maßnahmen der Dienstaufsicht sind daher gegenüber einem Richter nur zulässig, wenn und soweit sie keine Einflussnahme auf seine Rechtsanwendung enthalten (so im Fall einer dienstlichen Beurteilung BVerfG, Beschluss vom 15.6.1975, DRiZ 1975, 284).

c) Die richterliche Unabhängigkeit findet ihre Konkretisierung im Zusammenhang mit dem Prinzip der Gesetzesbindung des Richters (Art. 20 Abs. 3 GG). Das gilt in zweierlei Hinsicht: 
aa) Die Unterwerfung unter das Gesetz in Art. 97 Abs. 1 GG stellt klar, dass die richterliche Unabhängigkeit nicht für die Tätigkeit eines Richters gilt, der sich vorsätzlich in einer Art und Weise vom Gesetz entfernt, die den strafrechtlichen Tatbestand der Rechtsbeugung erfüllt (vgl. BVerfG, Beschluss vom 14.7.2016 - 2 BvR 661/16 -, zitiert nach Juris).

bb) Gleichzeitig ist die richterliche Unabhängigkeit eine entscheidende Bedingung für die Realisierung des rechtsstaatlichen Prinzips der Gesetzesbindung (BVerfG, Beschluss vom 14.7.2016 - 2 BvR 661/16 -, Rn. 17 sowie Rn. 20). Ohne richterliche Unabhängigkeit ist eine Realisierung der Gesetzesbindung in der Praxis der Rechtsanwendung nicht möglich. Eine Einflussnahme der Exekutive auf die Rechtsanwendung eines Richters ist - generell - gleichzeitig immer eine Einflussnahme im Sinne einer vollständigen oder teilweisen Lösung des Richters von seiner Gesetzesbindung.

Es gehört zum Wesen der Rechtsanwendung, dass es keine mathematische oder naturwissenschaftliche Methode gibt, zwischen „richtiger“" und „falscher" Gesetzesanwendung zu unterscheiden. Der Rechtsstaat akzeptiert, dass der einzelne Richter einerseits verpflichtet ist, das „richtige“ Recht zu „erkennen“, dass andererseits aber generell die Möglichkeit besteht, dass ein anderer Richter eine andere Rechtsanwendung als ,richtig“ erkennt. Jeder Richter entspricht seiner Pflicht zur Gesetzesbindung dadurch - und nur dadurch -, dass er sich entsprechend seinem Richtereid eine unabhängige richterliche Überzeugung davon bildet, was ,richtig“ ist, und sodann nur nach dieser Überzeugung entscheidet. Ein Richter der einer Einflussnahme der Exekutive nachgibt, und entgegen seiner richterlichen Überzeugung entscheidet, verletzt seine Pflicht zur Gesetzesbindung, und zwar unabhängig davon, ob andere Richter anders entscheiden würden, und unabhängig davon, ob man die rechtliche Überzeugung des Richters bei einer Betrachtung von außen als fehlerhaft einstufen würde. In letzter Konsequenz bedeutet das Nachgeben eines Richters gegenüber einem Ansinnen der Exekutive, dass der Richter, der überzeugungswidrig Recht anwendet, sich in den Bereich der strafbaren Rechtsbeugung begibt. Wenn die vom Richter missachtete eigene Überzeugung falsch war, ändert sich an der Strafbarkeit nichts; es würde nach allgemeinen strafrechtlichen Grundsätzen eine versuchte Rechtsbeugung bleiben.

Das bedeutet: Wenn die Exekutive einen Richter dazu drängt, seine Rechtsanwendung zu ändern und überzeugungswidrig Recht zu sprechen, dann drängt die Exekutive den Richter gleichzeitig in den Bereich einer versuchten oder vollendeten Rechtsbeugung. Der Beschwerdeführer hat den verfassungsrechtlichen Zusammenhang zwischen richterlicher Unabhängigkeit und Gesetzesbindung und die Bedeutung dieses Zusammenhangs für das dienstrechtliche Verfahren im Schriftsatz vom 27.11.2013 (Anlage 12, Ziff. 2 bis 8, S. 3 bis S. 15) ausführlich dargestellt und erläutert. Auf diese Ausführungen wird ergänzend verwiesen. 
d) Die richterliche Unabhängigkeit wird durch das Gebot des effektiven Rechtsschutzes (Art. 19 Abs. 4 GG) und durch Art. 6 Abs. 1 EMRK (Recht auf ein faires Verfahren, Entscheidung in angemessener Frist) weder begrenzt noch konkretisiert (unzutreffend daher die Begründung im Urteil des Dienstgerichts für Richter vom 4.12.2012, Anlage 4, S. 17, 18; unklar BGH im ersten Revisionsurteil vom 7.9.2017, Anlage 19, S. 12, 13).

e) Entscheidend ist dabei eine verfassungsrechtliche Vorfrage: Adressat der Garantien gemäß Art. 19 Abs. 4 GG und Art. 6 Abs. 1 EMRK ist nicht der einzelne Richter, sondern der Staat als Ganzes mit seinen verschiedenen Organen. Der Richter wird Adressat der Rechtsschutzgarantie im Einzelfall nur dann und nur insoweit, als er im Rahmen seiner verfassungsrechtlichen Stellung die Möglichkeit hat, auf die Effektivität des Rechtsschutzes für einen Bürger Einfluss zu nehmen. Es gibt im Grundgesetz weder eine Einschränkung der Gesetzesbindung durch das Gebot des effektiven Rechtsschutzes, noch gibt es nach dem Grundgesetz für den Richter und schon gar nicht für die Dienstaufsicht - ein Gebot oder eine Möglichkeit, zwischen Gesetzesbindung und effektivem Rechtsschutz abzuwägen. Wenn ein Richter nach seiner Überzeugung Zeit für die Rechtsanwendung braucht, dann ist er verpflichtet, diese Zeit aufzuwenden. Dem Richter ist es nicht gestattet, rechtliches Gehör zu verkürzen, nach seiner Überzeugung relevante Fragen nicht zu prüfen, oder eine Forderung für verjährt zu erklären, nur um ein bestimmtes Verfahren oder alle ihm zugewiesenen Verfahren - zu beschleunigen. Die Frage, ob und inwieweit bei der Auslegung bestimmter Gesetze - z. B. bei der Einzelrichterübertragung im Zivilprozess oder bei anderen Fragen der Verfahrensgestaltung im Rahmen der Zivilprozessordnung - Effizienzgesichtspunkte zu berücksichtigen sind, ist im Rechtsstaat eine Frage, die allein die zuständigen Richter in richterlicher Unabhängigkeit beantworten müssen, und nicht etwa eine Gerichtspräsidentin.

Wenn Gerichtsverfahren zu lange dauern, oder bestimmte Verfahren zeitweise liegen bleiben, weil der zuständige Richter zu viel Zeit für andere, vorrangige Verfahren benötigt, dann gibt es mögliche Ursachen bei verschiedenen Verfassungsorganen und Akteuren. Alle diese Akteure haben eine jeweils zu unterscheidende Verantwortung in ihrem jeweiligen Aufgabenbereich. Der Bundesgesetzgeber regelt mit Verfahrensregeln und mit materiellen Regeln die bindenden Vorgaben für die Richter bei der Rechtsanwendung. Die Legislative der Länder muss im Haushalt prüfen und entscheiden, welche Ressourcen für die Gerichte notwendig sind. Die Justizverwaltung hat die Aufgabe, notwendige organisatorische Voraussetzungen für einen effektiven Rechtsschutz zu schaffen und muss die Ressourcen verteilen. Die Gerichtspräsidien haben bei der gerichtsinternen Aufgabenverteilung das Gebot des effektiven Rechtsschutzes zu berücksichtigen. Wenn ein Richter auf Grund seiner Überzeugungen Zeit benötigt, dann verpflichtet ihn die Gesetzesbindung, diese Zeit aufzuwenden. Die Verantwortung für eine zu lange Verfahrensdauer liegt in diesem Fall bei den anderen staatlichen Akteuren.

Das Verhältnis zwischen Gesetzesbindung und richterlicher Unabhängigkeit einerseits und dem Gebot des effektiven Rechtsschutzes andererseits ist verfassungs- 
rechtlich eindeutig. Es gibt keine Entscheidung des Bundesverfassungsgerichts, die eine andere Sichtweise rechtfertigen könnte (vgl. für die Strafjustiz Landau, NStZ 2015, 665, 670). Wer zwischen richterlicher Unabhängigkeit und Gesetzesbindung auf der einen Seite und den Befugnissen der Dienstaufsicht auf der anderen Seite Abwägungen unter Zweckmäßigkeitsgesichtspunkten vornehmen möchte (so möglicherweise die Mehrzahl der Präsidentinnen und Präsidenten der Oberlandesgerichte in ihrer Stellungnahme vom 12.7.2017 zu einer Umfrage der Neuen Richtervereinigung, vgl. die Anlagen 33 und 34), verlässt den Bereich des Grundgesetzes. Wenn Richterinnen und Richter ihrer Überzeugung folgen (vgl. die Fragen der Neuen Richtervereinigung in der Anlage 33), gibt es das von den Präsidentinnen und Präsidenten angenommene ,Spannungsverhältnis zwischen richterlicher Unabhängigkeit, gesetzlichem Beschleunigungsgebot, sowie Verantwortung und Grenzen der Dienstaufsicht" (vgl. die Anlage 34) aus der Perspektive des Grundgesetzes nicht.

\section{Die Verletzung der richterlichen Unabhängigkeit im Urteil des Bundesge- richtshofs vom 12.5.2020}

a) Die Argumentation in der angegriffenen Entscheidung entspricht in wesentlichen Elementen nicht den verfassungsrechtlichen Anforderungen gemäß Art. 97 Abs. 1 GG. Die Entscheidung verkennt zum einen Inhalt und Bedeutung von Art.97 Abs. 1 GG; zum anderen wird der streitgegenständliche Sachverhalt nicht subsumiert (siehe im Einzelnen die folgenden Ausführungen). Bei einer korrekten Anwendung von Art. 97 Abs. 1 GG hätte der Bundesgerichtshof auf die Revision des Beschwerdeführers die Entscheidung des Dienstgerichtshofs und das erstinstanzliche Urteil aufheben und entsprechend des Antrags des Beschwerdeführers die angegriffene Maßnahme der Präsidentin im Bescheid vom 26.1.2012 wegen Verletzung der richterlichen Unabhängigkeit für unzulässig erklären müssen.

b) Die Präsidentin des Oberlandesgerichts hat in einem von ihr selbst entworfenen und unterzeichneten Schriftsatz vom 29.4.2013 (Anlage 7, S. 3) vor dem Dienstgerichtshof Inhalt und Ziel der Maßnahme vom 26.1.2012 wie folgt konkretisiert:

\section{„Durch die gesetzliche Vorgabe der Personalausstattung und das tatsächliche Fallaufkommen wird aber der - auch für den Berufungsführer - verbindliche Maßstab aufgestellt, wie viel der einzelne Richter in seiner jeweiligen Funktion insgesamt zu erledigen hat. “"}

Das ist der Kern der Maßnahme der Präsidentin. Es geht um ein politisches Ziel der baden-württembergischen Justizverwaltung, für das es keine Rechtsgrundlage gibt. Die politische Zielvorstellung der Präsidentin widerspricht sowohl der richterlichen Unabhängigkeit, als auch der Gesetzesbindung des Richters, der sich nach materiellem und formellen Recht Bundesrecht zu richten hat, und nicht nach den Haushaltsvorstellungen des Landesgesetzgebers. Der Bundesgerichtshof hat die von der Präsidentin formulierte Zielvorstellung in der angegriffenen Entscheidung nicht erwähnt und nicht berücksichtigt, obwohl der Beschwerdeführer wiederholt auf die Bedeutung dieser Erklärung hingewiesen hat. Der Bundesgerichts- 
hof hat Art. 97 Abs. 1 GG auch dadurch verletzt, dass er die eigene Interpretation der Maßnahme vom 26.1.2012 durch die Präsidentin übergangen hat.

c) Die Entscheidung des Bundesgerichtshofs unterlässt eine Subsumtion des streitgegenständlichen Sachverhalts.

Streitgegenstand war der Inhalt des Bescheids vom 26.1.2012. Mit den Formulierungen in diesem Bescheid hat die Präsidentin den Beschwerdeführer aufgefordert, seine Rechtsanwendung grundlegend zu ändern, um die von der Präsidentin verlangten Durchschnittszahlen zu erzielen. Dass dies der für den Beschwerdeführer ersichtliche Inhalt des Bescheids sein sollte, hat die Präsidentin in dem zitierten Schriftsatz vom 29.4.2013 selbst eingeräumt (siehe oben b)). Aus einem ausführlichen Gespräch vom 30.4.2010 waren der Präsidentin die Ursachen für die unterdurchschnittlichen Erledigungszahlen des Beschwerdeführers bekannt (vgl. den im Ausgangsverfahren vorgelegten und inhaltlich unstreitigen Vermerk vom 6.11.2011, Anlage 8). Eine andere Möglichkeit zur Erzielung höherer Erledigungszahlen hat die Präsidentin dem Beschwerdeführer zudem unstreitig nie genannt. Der Bundesgerichtshof hat den Kern des Sachverhalts im Tatbestand und in den Entscheidungsgründen des Urteils übergangen. (Weitere in diesem Zusammenhang vom Bundesgerichtshof nicht berücksichtigte Umstände werden im Einzelnen unten bei der Verletzung des rechtlichen Gehörs unter VI. im Einzelnen dargestellt.)

d) Tragender Grund der Entscheidung des Bundesgerichtshofs ist die Erwägung, die Dienstaufsicht dürfe gegenüber einem Richter bei Rückständen und unterdurchschnittlichen Erledigungszahlen nur dann nicht einschreiten, ,wenn dem Richter damit indirekt ein Pensum abverlangt wird, welches sich allgemein, also auch von anderen Richtern, sachgerecht nicht mehr bewältigen lässt" (Urteil des Bundesgerichtshofs vom 12.5.2020, Anlage 2, Rn. 28). Diese Erwägung verlässt den Boden des Grundgesetzes.

aa) Richter arbeiten in der Rechtsanwendung generell unterschiedlich mit - gerade am Oberlandesgericht - sehr unterschiedlichem Zeitbedarf pro Fall. Die Forderung des Bundesgerichtshofs würde zum einen bedeuten, dass Richter eine „Durchschnittsachgerechtigkeit“ in der Rechtsanwendung anderer Richter zum Maßstab ihrer eigenen Rechtsprechung erheben müssten. Zum anderen würde die Forderung des Bundesgerichtshofs bedeuten - wenn sie richtig wäre -, dass die Exekutive in Gestalt der Justizverwaltung die Einhaltung der „Durchschnittssachgerechtigkeit" in der Arbeit der Richter kontrollieren und bei Abweichungen sanktionieren dürfte.

bb) Die Begriffsbildung des Bundesgerichtshofs entspricht nicht dem Grundsatz der richterlichen Unabhängigkeit, der vom Bundesgerichtshof zu prüfen war (siehe oben V. 1.). Es gibt keine Entscheidung des Bundesverfassungsgerichts, die der Prüfung einer vermeintlichen „Sachgerechtigkeit“ im Rahmen von Art. 97 Abs. 1 GG eine Bedeutung zuweisen würde. 
cc) Der Bundesgerichtshof nimmt zum Maßstab der „Sachgerechtigkeit“ Bezug auf eigene Entscheidungen (Urteil vom 12.5.2020, Anlage 2, Rn. 28, 38, sowie das Urteil vom 7.9.2017, Anlage 19 Rn. 24), die ähnliche Formulierungen zur „Sachgerechtigkeit“ im Zusammenhang mit einer kritischen Bewertung von Erledigungszahlen enthalten. Die zitierten Formulierungen in den früheren Entscheidungen waren allerdings unklar. Sie wären verfassungsrechtlich nur dann haltbar gewesen, wenn jeder Richter gemäß seiner eigenen Überzeugung entscheiden sollte, was für ihn in der Rechtsanwendung "sachgerecht" ist. Die Formulierungen sind jedoch wegen Verstoßes gegen Art. 97 Abs. 1 GG verfassungswidrig, wenn eine „sachgerechte“ Durchschnittsrechtsprechung anderer Richterinnen und Richter am Oberlandesgericht Karlsruhe zum Maßstab der von der Dienstaufsicht verlangten Rechtsanwendung des Beschwerdeführers werden soll. Dass die Formulierungen im letzteren (verfassungswidrigen) Sinne gemeint sein sollen, hat der Bundesgerichtshof in der angegriffenen Entscheidung (Anlage 2, Rn. 38) und in der Entscheidung vom 7.9.2017 (Anlage 19, Rn. 28 ff.) klargestellt. (Vgl. zur Analyse der BGH-Rechtsprechung die Ausführungen des Beschwerdeführers in der Revisionsbegründung vom 7.10.2019, Anlage 27, und in der früheren Revisionsbegründung vom 23.9.2015, Anlage 16, S. 22 f. sowie die Ausführungen im Plädoyer vom 12.5.2020, Anlage 28, S. 8 ff.).

dd) Im angegriffenen Urteil des Bundesgerichtshofs finden sich längere Ausführungen zur Ermittlung der Sachgerechtigkeit durch die Präsidentin des Oberlandesgerichts (Anlage 2, Rn. 32 ff.). Diese Ausführungen sind nicht erheblich. Der Beschwerdeführer hat im dienstgerichtlichen Verfahren nie bestritten, dass auch alle anderen Richterinnen und Richter am Oberlandesgericht - in ihrer jeweils unterschiedlichen Rechtsanwendung - nach ihrer eigenen Überzeugung und daher ,sachgerecht“" arbeiten.

ee) Der Bundesgerichtshof hat in der Entscheidung vom 12.5.2020 gefordert, dass Richterinnen und Richter in Deutschland den Maßstab der „Sachgerechtigkeit“, der sich nach einem „Durchschnitt“ aller Richter bemessen solle, über ihre richterliche Überzeugung und über ihre Pflicht zur (überzeugungsgemäßen) Gesetzesbindung stellen müssen. Und der Bundesgerichtshof hat darüber hinaus postuliert, dass die Justizverwaltung den Vorrang der „Sachgerechtigkeit“ gegenüber der Gesetzesbindung der Richter durch Maßnahmen der Dienstaufsicht kontrollieren und sicherstellen dürfe. Die gleichzeitige widersprüchliche Leugnung dieser Konsequenz im angegriffenen Urteil (Anlage 2, Rn. 38) ist nicht nachvollziehbar. Eine Kontrolle einer solchen „Sachgerechtigkeit“ der Rechtsprechung durch die Exekutive ist im Rechtsstaat ausgeschlossen.

e) Der Vorhalt einer „unzureichenden Arbeitsleistung“ und eine damit verbundene Ermahnung sind nur dann und nur insoweit zulässig, als damit keine Einflussnahme (auch keine indirekte, subtile oder psychologische Einflussnahme) verbunden ist (s. o. V. 1.). Das könnte im Einzelfall beispielsweise möglich sein bei einer unzureichenden Arbeitszeit eines Richters, bei einer Weigerung eines Richters, ein 
bestimmtes ihm zugewiesenes Verfahren zu bearbeiten, oder evtl. bei der willkürlichen Nichtbeachtung einer gesetzlichen Frist. Solche Umstände liegen im Fall des Beschwerdeführers nicht vor und sind vom Bundesgerichtshof auch nicht angenommen worden. Soweit die Entscheidung (Anlage 2, Rn. 25 ff.) behauptet, die Maßnahme der Präsidentin vom 26.1.2012 (Anlage 5) habe keinen Einfluss genommen, handelt es sich um Leerformeln, die den allgemeinen Denkgesetzen und dem Vorbringen der Parteien widersprechen (siehe auch die Ausführungen unten g), h) und VI. 1.).

Es gibt keine Entscheidung des Bundesverfassungsgerichts, welche die Verkürzung von Art. 97 Abs. 1 GG durch den Bundesgerichtshof rechtfertigen würde. Insbesondere hat das Bundesverfassungsgericht zu keinem Zeitpunkt entschieden, Richter seien generell verpflichtet, ein in Erledigungszahlen zu messendes Arbeitspensum zu leisten. Die Formulierung des Bundesverfassungsgerichts im Beschluss vom 23.5.2012 - 2 BvR 610/12, 2 BvR 625/12 - (Rn. 17, zitiert nach Juris) zur Frage eines „Arbeitspensums“ von Richtern geht auf Entscheidungen des Bundesverwaltungsgerichts zurück, in denen es um die Frage ging, inwieweit Richter verpflichtet sind, bestimmte Dienstzeiten und bestimmte Arbeitszeiten einzuhalten. Dementsprechend geht es auch in der Entscheidung des Bundesverfassungsgerichts vom 23.5.2012 nur um Fragen zur verpflichtenden Arbeitszeit von Richtern, und nicht um eine Inhaltsbestimmung, Verkürzung oder Beschränkung der richterlichen Unabhängigkeit durch ein zahlenmäßig definiertes „Arbeitspensum“. In einer Entscheidung vom 25.2.1964 - 2 BvR 411/61 - hat das Bundesverfassungsgericht zudem ausdrücklich darauf hingewiesen, dass ein unterschiedlicher Zeitbedarf von Richtern - entgegen der Auffassung des Bundesgerichtshofs in der angegriffenen Entscheidung - zum Alltag eines Gerichts gehört, mit der möglichen Konsequenz unterschiedlicher Erledigungszahlen (vgl. BVerfG, Beschluss vom 25. Februar 1964 - 2 BvR 411/61 -, Rn. 15, zitiert nach Juris; im entschiedenen Fall wegen generell zu erwartender unterschiedlicher Leistungsfähigkeit von Richtern).

f) Der Bundesgerichtshof missachtet die neuere Rechtsprechung des Bundesverfassungsgerichts. Das Bundesverfassungsgericht hat in einer Entscheidung vom 14.7.2016 - 2 BvR 661/16 - (Rz. 20, 21) die Bedeutung der zur Verfügung stehenden Zeit für die Rechtsanwendung des Richters und für die richterliche Unabhängigkeit hervorgehoben:

„(...) Die Verwirklichung dieser Zielsetzung setzt jedoch voraus, dass dem zur Entscheidung berufenen Richter ausreichend Zeit zu einer allein an Gesetz und Recht orientierten Bearbeitung des Falles zur Verfügung steht. Nur wenn dies gewährleistet ist, kann der Richter seiner persönlichen Verantwortung gerecht werden. Dabei wird stets die konkrete, subjektive Belastungssituation des Richters in den Blick zu nehmen sein. Eine Orientierung an vermeintlich objektiven, durchschnittlichen Bearbeitungszeiten genügt dem nicht." 
Und weiter:

„Insoweit hat das Bundesverfassungsgericht bereits festgestellt, dass das gegenwärtige System der Bewertung richterlicher Arbeit nicht unwesentlich nach quantitativen Gesichtspunkten erfolgt und hierdurch zusätzliche Anreize für eine möglichst rasche Verfahrenserledigung unter Inkaufnahme inhaltlicher Defizite schafft (vgl. BVerfGE 133, 168, 177 Rn. 3).“

Der Beschwerdeführer hat auf diese verfassungsrechtlichen Ausführungen im dienstgerichtlichen Verfahren hingewiesen, und zwar zunächst in einem Schriftsatz vom 8.9.2016, der ausschließlich die zitierte Entscheidung des Bundesverfassungsgerichts zum Gegenstand hatte, und sodann nochmals im Plädoyer im Termin zur ersten mündlichen Revisionsverhandlung (Anlage 18, S. 13). Die Entscheidung des Bundesverfassungsgerichts wird weder in der früheren Revisionsentscheidung noch in der angegriffenen Entscheidung des Bundesgerichtshofs vom 12.5.2020 erwähnt. Dementsprechend hat sich der Bundesgerichthof mit der Entscheidung des Bundesverfassungsgerichts vom 14.7.2016 auch nicht auseinandergesetzt.

g) Der Bundesgerichtshof behauptet in der früheren Entscheidung vom 17.9.2017 (Anlage 19, Rn. 25), es sei unzutreffend, dass nur „strukturelle Langsamkeit oder überdurchschnittliche Sorgfalt" ursächlich für unterdurchschnittliche Erledigungszahlen des Beschwerdeführers sein könnten; es könnten auch ,andere Ursachen“ maßgeblich sein. Die Behauptung des Bundesgerichtshofs widerspricht den Denkgesetzen. Denn sowohl der Bundesgerichtshof als auch die Vorinstanzen haben ebenso wie die Präsidentin des Oberlandesgerichts und die Vertreter der Antragsgegnerseite im Ausgangsverfahren - dem Beschwerdeführer in den letzten acht Jahren nie erklärt, welche Möglichkeiten - außer einer Änderung der Rechtsanwendung - zur Steigerung von Erledigungszahlen für ihn in Betracht kommen sollen. Die Behauptung der Präsidentin, der Vorhalt und die Ermahnung zur Erzielung von Durchschnittszahlen im Bescheid vom 26.012012 hätten nichts mit der Rechtsanwendung des Beschwerdeführers zu tun, hat Fabian Wittreck (Anlage 35) schon 2012 aus tatsächlichen Gründen als perfide und perplex bezeichnet (NJW 2012, 3287, 3290). Sie widerspricht zudem der eigenen Erklärung der Präsidentin zu der angeblichen ,gesetzlichen Vorgabe der Personalausstattung“. Am Rande ist darauf hinzuweisen, dass der Beschwerdeführer in den dienstgerichtlichen Verfahren keine „überdurchschnittliche Sorgfalt“ für sich in Anspruch genommen hat, sondern nur eine seiner richterlichen Überzeugung entsprechende Rechtsanwendung (vgl. insbesondere den Schriftsatz vom 27.11.2013, Anlage 12, S. 4 f. und S. 8 sowie den als Anlage $\mathrm{zu}$ diesem Schriftsatz vorgelegten Vermerk vom 6.11.2011, Anlage 8).

Der Bundesgerichtshof hat bei der zitierten Behauptung zudem wesentliche Teile des Sachverhalts und des Sachvortrags übergangen. Auf diese Fehler wird unten gesondert eingegangen (VI. 1.).

h) Die angegriffene Entscheidung beschäftigt sich in rechtlicher Hinsicht an keiner Stelle mit der Frage, welche Bedeutung die jeweilige richterliche Überzeugung im Rahmen von Art. 97 Abs. 1 GG haben muss. Der Hinweis des Bundesgerichtshofs auf „subjektive Vorstellungen des einzelnen Richters“ (Urteil vom 7.9.2017, Anla- 
ge 19, Rn. 22) verkennt die verfassungsrechtliche Bedeutung der richterlichen Überzeugung. Die Bedeutung der richterlichen Überzeugung für den Zeitbedarf des einzelnen Richters war sowohl vor dem Dienstgerichtshof als auch in der Revisionsinstanz ein zentraler Punkt im Vorbringen des Beschwerdeführers (vgl. den Schriftsatz vor dem Dienstgerichtshof, Anlage 12, S. 3 ff. und die erste Revisionsbegründung, Anlage 16, S. 22). Ebenso fehlt eine Auseinandersetzung mit der Gesetzesbindung des Richters, die mit der richterlichen Unabhängigkeit korrespondiert. Nur mit einer Berücksichtigung von richterlicher Überzeugung und Gesetzesbindung hätte der Bundesgerichtshof zu einem verfassungsrechtlich zutreffenden Ergebnis bei der Frage gelangen können, was der Zeitbedarf eines Richters im Rahmen der richterlichen Unabhängigkeit bedeuten muss. Die Entscheidung berücksichtigt nicht, dass der Beschwerdeführer bei einer überzeugungswidrigen Rechtsanwendung in den Bereich der versuchten oder vollendeten Rechtsbeugung gelangen würde (siehe oben V. 1. c)).

i) Die erste Entscheidung des Bundesgerichtshofs vom 7.9.2017 führt aus (Anlage 19, Rn. 18), es sei zu differenzieren, ob eine Maßnahme der Dienstaufsicht einerseits die „Sicherung eines ordnungsgemäßen Geschäftsablaufs“ oder andererseits den „Kernbereich“ der Rechtsprechung betreffe. Eine solche Unterscheidung - die in der dienstgerichtlichen Rechtsprechung des Bundesgerichtshofs in den vergangenen Jahrzehnten entwickelt wurde - ergibt sich weder aus Art. 97 Abs. 1 GG noch aus der Rechtsprechung des Bundesverfassungsgerichts. Die Entscheidungen des Bundesverfassungsgerichts zur richterlichen Unabhängigkeit kennen eine solche Differenzierung nicht.

Gemäß Art. 97 Abs. 1 GG und nach der Rechtsprechung des Bundesverfassungsgerichts kommt es allein auf die Sachverhaltsfrage an, ob eine bestimmte Maßnahme der Exekutive Einfluss auf die Rechtsanwendung eines Richters nimmt, bzw., ob nach allgemeiner Erfahrung davon auszugehen ist, dass eine bestimmte Maßnahme einen solchen Einfluss haben kann (siehe oben V. 1. und die dort zitierte Rechtsprechung des Bundesverfassungsgerichts). Der Bundesgerichtshof hat die sogenannte Kernbereichslehre ursprünglich im Sinne einer bloßen Etikettierung entwickelt. Wenn eine Maßnahme der Exekutive Auswirkungen auf die Rechtsanwendung eines Richters haben kann, wird die Maßnahme dem „Kernbereich“ zugewiesen. Hat eine organisatorische Maßnahme hingegen offensichtlich keine Auswirkungen auf die Rechtsanwendung (z. B. Zuweisung eines bestimmten Sitzungssaals oder Zuweisung einer bestimmten Mitarbeiterin), erhält sie in der Rechtsprechung der Dienstgerichte das Etikett ,äußere Ordnung“. Gegen eine solche sprachliche Differenzierung (Etikettierung) ist nichts einzuwenden. Wenn hingegen der Begriff der ,äußeren Ordnung“ oder des „ordnungsgemäßen Geschäftsablaufs“ ohne Prüfung der möglichen Einflussnahme auf die Rechtsanwendung gebildet wird (so muss man wohl die Formulierungen des Bundesgerichtshofs in der Entscheidung vom 7.9.2017 Anlage 19, Rn. 18, verstehen), ist dies mit Art. 97 Abs. 1 GG und mit der Rechtsprechung des Bundesverfassungsgerichts nicht vereinbar. 
j) Die Verfassungsbeschwerde betrifft eine Einwirkung der Justizverwaltung auf die Rechtsanwendung des Beschwerdeführers durch Vorhalt und Ermahnung. Die Verfassungsbeschwerde betrifft hingegen nicht die davon $\mathrm{zu}$ trennende Frage einer eventuellen begrenzten psychologischen Einflussnahme auf die Tätigkeit der Richterinnen und Richter durch Formulierungen in dienstlichen Beurteilungen. Soweit ersichtlich hat das Dienstgericht des Bundes die Möglichkeit einer Differenzierung bei Beeinträchtigungen durch Formulierungen in dienstlichen Beurteilungen in den Entscheidungen der vergangenen Jahrzehnte nicht erörtert (anders Wittreck, NJW 2012, 3287 ff. und Wittreck, DRiZ 2013, 60 f.). Da das Grundgesetz die Organisation der Justiz - einschließlich der Organisation der Einstellung und Beförderung von Richtern - der Exekutive in Bund und Ländern zuweist, liegen Differenzierungen bei dienstlichen Beurteilungen, soweit sie durch die Organisation der Justiz geboten sind, verfassungsrechtlich nicht fern. Wenn eine dienstliche Beurteilung sich auch zur Frage der Arbeitsmenge eines Richters äußert, und dabei in gewissem Umfang auch sachliche und vernünftige Informationen zu Fallzahlen und Erledigungszahlen enthält, ohne diese mit einer Handlungsaufforderung an den Richter zu verbinden, dann dürfte dies verfassungsrechtlich kaum zu beanstanden sein.

\section{Begründetheit - Verletzung des rechtlichen Gehörs (Art.103 Abs. 1 GG)}

Die Entscheidung des Bundesgerichtshofs verletzt das rechtliche Gehör des Beschwerdeführers (Art. 103 Abs. 1 GG).

1. Wesentliche Teile des Sachverhalts und des Beschwerdeführer-Vorbringens sind sowohl vom Dienstgerichtshof als auch vom Bundesgerichtshof unter Verletzung des rechtlichen Gehörs nicht berücksichtigt worden. Es geht in der Revisionsbegründung um die folgenden Hinweise auf unstreitigen Sachverhalt bzw. um Rügen der Sachverhaltserfassung durch den Dienstgerichtshof, die der Bundesgerichtshof übergangen hat:

a) Es gibt außer einer Änderung der Rechtsanwendung in einer Vielzahl von Fällen keine andere Möglichkeit, wie der Beschwerdeführer dem Ansinnen der Präsidentin zur Erzielung höherer Erledigungszahlen Folge leisten könnte (vgl. die Revisionsbegründung vom 7.10.2019, Anlage 27 [im Folgenden abgekürzt: RB], S. 8 f., S. 13 f., im Berufungsverfahren zu Unrecht abgelehnter Beweisantrag I. 5., RB, S. $16-17$ ).

b) Die Präsidentin hat ihre Zielsetzung im Verfahren vor dem Dienstgerichtshof selbst wie folgt formuliert: „Durch die gesetzliche Vorgabe der Personalausstattung und das tatsächliche Fallaufkommen wird aber der - auch für den Berufungsführer - verbindliche Maßstab aufgestellt, wie viel der einzelne Richter in seiner jeweiligen Funktion insgesamt zu erledigen hat." (RB S. 6 und S. 10, Anlage 7, S. 3). 
c) Die Zeit pro Fall, die ein Richter benötigt, hängt generell in erheblichem Umfang von der individuellen (überzeugungsgemäßen) Rechtsanwendung ab (Anlage 12, S. 4 ff, vorgelegt im Revisionsverfahren als Anlage R 3 zur RB).

d) Aus dem Gespräch vom 30.4.2010, das durch einen unstreitigen Vermerk vom 6.11.2011 dokumentiert ist (Anlage 8, im Ausgangsverfahren sowohl vor dem Dienstgerichtshof als auch im Revisionsverfahren jeweils vorgelegt), ergaben sich für die Präsidentin die Details der Arbeitsweise des Beschwerdeführers, die in der Rechtsanwendung zu einem höheren Zeitbedarf führen (RB S. 9 und S. 14).

e) Es entsprach dem Vorsatz und dem Willen der Präsidentin, den Beschwerdeführer mit dem Bescheid vom 26.1.2012 unter Druck zu setzen, damit er seine Rechtsanwendung ändert (RB S. 10 und S. 14).

f) Die Präsidentin kannte aus verschiedenen (vom Beschwerdeführer konkretisierten) Umständen die allein in der Rechtsanwendung des Beschwerdeführers liegenden Ursachen für seinen Zeitbedarf (vgl. RB S. 14 und RB S. 18 f.).

g) Täuschungsversuche der Gegenseite in der Prozessführung des Parallelverfahrens (wegen der Sonderprüfung) sind ein Indiz für die Zielrichtung des Bescheids vom 26.1.2012 (im Berufungsverfahren zu Unrecht abgelehnte Beweisanträge IV. Ziff. 4, 5, 6 und 7, RB 19, 20).

h) Der auf einer unterschiedlichen Arbeitsweise (Rechtsanwendung) beruhende Zeitbedarf der Richter am Oberlandesgericht differiert nicht selten um 300 Prozent; das heißt, es gibt am Oberlandesgericht Richter die in der gleichen Zeit viermal so viele Fälle erledigen können wie andere Richter (Beweisanträge im Berufungsverfahren I. Ziff. 1 und Ziff. 2 als wahr unterstellt, RB S. 11 f. und S. 14; jedoch weder vom Dienstgerichtshof noch vom Bundesgerichtshof berücksichtigt).

i) Es gibt eine größere Anzahl von Indizien für ein willkürliches Handeln der Präsidentin (RB S. 13 f,).

2. Die unter 1. dargestellten Umstände waren für die Entscheidung des Bundesgerichtshofs erheblich, wenn man die Rechtsauffassung des Bundesgerichtshofs zu Grunde legt. Die angegriffene Entscheidung leugnet eine sich bereits unmittelbar aus dem Bescheid vom 26.1.2012 ergebende Einflussnahme auf die Rechtsanwendung des Beschwerdeführers. Wenn man dies unterstellt, hätten für den Bundesgerichtshof alle relevanten Umstände des Sachverhalts, die Gegenstand des Verfahrens waren, bzw. die vom Dienstgerichtshof unter Verletzung von Verfahrensvorschriften übergangen worden waren, Anlass für die Prüfung sein müssen, ob sich aus diesen - vom Beschwerdeführer in der Revisionsbegründung hervorgehobenen - Umständen eine Zielrichtung der Maßnahme der Präsidentin im Sinne einer Einwirkung auf die Rechtsanwendung des Beschwerdeführers ergeben kann. Bei 
allen oben unter 1. aufgeführten Umständen ergibt sich aus logischen Gründen: Ein Teil der Umstände führt zwingend zu dem Schluss, dass die Maßnahme der Präsidentin vom 26.1.2012 eine generelle Änderung der Rechtsanwendung des Beschwerdeführers bewirken sollte; ein anderer Teil der Umstände hat zumindest indiziellen Charakter in diesem Sinne.

3. Gemäß Art. 103 Abs. 1 GG hätte der Bundesgerichtshof auf der Basis seines Entscheidungskonzepts sämtliche der oben 1. aufgeführten Umstände bzw. Rügen berücksichtigen müssen. Dies ist ausweislich der Entscheidungsgründe jedoch nicht erfolgt. Daraus ergibt sich die Verletzung von Art. 103 Abs. 1 GG.

Die Pflicht des Gerichts zur Berücksichtigung von entscheidungserheblichem Vorbringen ist wesentlicher Teil des Grundrechts gemäß Art. 103 Abs. 1 GG. Zwar muss nicht jedes Vorbringen eines Verfahrensbeteiligten in den Gründen einer Gerichtsentscheidung ausdrücklich beschieden werden. Jedoch sind die wesentlichen Tatsachenbehauptungen in den Entscheidungsgründen zu verbescheiden. Auch bei einer Revisionsentscheidung ist von einem Schweigen zu einem bestimmten Vorbringen auf eine Nichtberücksichtigung zu schließen, wenn bestimmte Umstände dies nahelegen (vgl. BVerfG, Urteil vom 8.7.1997 - 1 BvR 1621/94 -, Rn. 44, 45, zitiert nach Juris; BVerfG, Beschluss vom 25.10.2011 - 2 BvR 2407/10 -, Rn. 5 ff., zitiert nach Juris).

Vorliegend ergibt sich die Nichtberücksichtigung aus dem Inhalt der vom Beschwerdeführer vorgebrachten Umstände. Die Auffassung des Bundesgerichtshofs, die Maßnahme der Präsidentin vom 26.1.2012 hätte keinen Bezug zur Rechtsanwendung des Beschwerdeführers gehabt, ist unter keinem denkbaren Gesichtspunkt mit den unter oben 1. aufgeführten Umständen vereinbar. Es ist insbesondere nicht erkennbar, wie der Beschwerdeführer höhere Erledigungszahlen ohne Änderung seiner Rechtsanwendung erzielen soll (oben 1. a)), wenn niemand eine andere Möglichkeit nennt. Es widerspricht der Vernunft, dem Bescheid vom 26.1.2012 ein anderes Ziel zuzusprechen, wenn die Präsidentin selbst ausführt, dass der Beschwerdeführer seine Rechtsanwendung der „gesetzlichen Vorgabe der Personalausstattung“" anpassen sollte (oben 1. b)). Es ist nicht erkennbar, was die Maßnahme der Präsidentin - außer einer Änderung der Rechtsanwendung des Beschwerdeführers - bewirken sollte, wenn sie die Ursachen seiner unterdurchschnittlichen Erledigungszahlen genau kannte (oben 1. d) und f)). Wenn die Präsidentin den Vorsatz und den Willen hatte, den Beschwerdeführer unter Druck zu setzen, damit er seine Rechtsanwendung ändert (oben 1. e)), ist nicht ersichtlich, wie der Bescheid vom 26.1.2012 für den Beschwerdeführer eine vom Vorsatz der Präsidentin abweichende Bedeutung haben könnte. Die Behauptung des Bundesgerichtshofs im angegriffenen Urteil (Anlage 2, Rn. 41) eine (für jedermann erkennbare) Absicht der Präsidentin könne keine Rolle für das Verständnis des Bescheids vom 26.1.2012 spielen, ist vernunftwidrig. Es sind schlechterdings keine sachlichen Gesichtspunkte denkbar, wie eine Berücksichtigung der oben 1. dargestellten Umstände bzw. Rügen mit der Entscheidungsbegründung des BGH ver- 
einbar sein könnte. Daraus ergibt sich der Schluss auf eine Verletzung von Art. 103 Abs. 1 GG.

4. Die Revision hätte bereits aus den oben V. angeführten verfassungsrechtlichen Gründen wegen Verletzung der richterlichen Unabhängigkeit Erfolg haben müssen. Auf der Grundlage des vom BGH gewählten abweichenden Entscheidungskonzepts hätte jedoch auch eine Berücksichtigung des außer Acht gelassenen Vorbringens des Beschwerdeführers zu einer Revisionsentscheidung zu seinen Gunsten führen müssen. Die angegriffene Entscheidung beruht daher - auch - auf der Verletzung des rechtlichen Gehörs.

5. Der Beschwerdeführer hat die Verletzung des rechtlichen Gehörs vor der Verfassungsbeschwerde zum Gegenstand der Anhörungsrüge (Anlage 29) gemacht. Das vom Bundesgerichtshof nicht berücksichtigte Vorbringen des Beschwerdeführers wurde in der Anhörungsrüge bezeichnet. Die zulässige Anhörungsrüge wurde vom Bundesgerichtshof als unbegründet zurückgewiesen (Anlage 30).

\section{Begründetheit - Verletzung des Willkürverbots (Art.3 Abs 1 GG)}

Die Entscheidung des Bundesgerichtshofs verletzt den Beschwerdeführer in seinem Grundrecht aus Art. 3 Abs. 1 GG (Willkürverbot). Willkürlich ist ein Richterspruch, der unter keinem denkbaren Aspekt rechtlich vertretbar ist, so dass sich der Schluss aufdrängt, dass er auf sachfremden Erwägungen beruht. Dies ist an Hand objektiver Kriterien festzustellen (vgl. BVerfG, NJW 2005, 1999, 2003, 2004). Die angegriffene Entscheidung überschreitet aus mehreren Gründen die Grenzen der Willkür.

1. Die Ausführungen des Bundesgerichtshofs zum Begriff der richterlichen Unabhängigkeit sind im entscheidenden Punkt willkürlich. Es gibt keine Rechtfertigung für die Dienstaufsicht, Richter zu ermahnen, ein nach Zahlen bemessenes Durchschnittspensum zu erledigen. Es ist unter keinem denkbaren rechtlichen Aspekt vertretbar, eine Einflussnahme der Dienstaufsicht auf die Rechtsanwendung eines Richters zu gestatten, wenn es um Erledigungszahlen geht, bzw. um die Berücksichtigung einer „Vorgabe der Personalausstattung“ bei der Rechtsanwendung.

Die Frage der Gesetzesbindung und der richterlichen Unabhängigkeit im Zusammenhang mit „Arbeitspensen“" und Erledigungszahlen ist durch die Entscheidung des Bundesverfassungsgerichts vom 14.7.2016 - 2 BvR 661/16 -, dort Rz. 20, 21, konkretisiert worden. Obwohl die Bedeutung der Entscheidung auf der Hand liegt und der Beschwerdeführer auf die Entscheidung hingewiesen hat, wird diese Entscheidung des Bundesverfassungsgerichts in den Gründen der angegriffenen Entscheidung nicht erwähnt. Dies legt sachfremde Erwägungen des Bundesgerichtshofs nahe. 
Die Ausführungen des Bundesgerichtshofs zur angeblichen Bedeutung einer „Sachgerechtigkeit“" bei der Ermittlung von Durchschnittszahlen, die zum Maßstab für ein richterliches „Erledigungspensum“ werden sollen, lassen jede vernünftige Überlegung vermissen (s. o. V. 2. d)). Die Ausführungen haben keinen gedanklichen Bezug zum verfassungsrechtlichen Gehalt von Art. 97 Abs. 1 GG.

2. Zur richterlichen Unabhängigkeit gehört die Verpflichtung des Richters zur überzeugungsgemäßen Rechtsanwendung. Es liegt auf der Hand und ist jedem Juristen bekannt, dass eine überzeugungsgemäße Rechtsanwendung verschiedener Richter unterschiedlich ausfällt, und mit deutlich unterschiedlichem Zeitbedarf verbunden sein kann. In den Schriftsätzen des Beschwerdeführers im Ausgansverfahren ist das ein zentraler Punkt der Argumentation. Es ist nicht nachvollziehbar, dass sich der Bundesgerichtshof mit dieser Problematik im Rahmen von Art. 97 Abs. 1 GG nicht beschäftigt.

3. Der Umgang des Bundesgerichtshofs mit Inhalt und Bedeutung der Maßnahme vom 26.1.2012 ist angesichts des Sachverhalts, der dem Revisionsgericht vorlag, nicht erklärbar. Es wird auf die obigen Ausführungen (V. 2. b), c), g) und VI.) hierzu verwiesen. Ergänzend: Der Bundesgerichtshof kennt zwar den Aufsatz von Wittreck aus dem Jahr 2012 (Anlage 35; vgl. das Zitat im Urteil des BGH vom 7.9.2017, Anlage 19, Rn. 25); der entscheidende Punkt in diesem Aufsatz, nämlich die Wahrnehmung des Sachverhalts, wonach die Leugnung von Inhalt und Ziel des Bescheids vom 26.1.2012 „perfide“ und „perplex“ sei (Wittreck, NJW 2012, 3287, 3290), wird vom Bundesgerichtshof jedoch nicht erwähnt. Auch hier drängt sich der Eindruck sachfremder Erwägungen auf.

\section{Die Grundrechtsverstöße des Dienstgerichtshofs}

Das Urteil des Dienstgerichtshofs (Anlage 3) verletzt den Beschwerdeführer in gleicher Weise in seinen Grundrechten wie die angegriffene Entscheidung des Bundesgerichtshofs. Auf die Ausführungen zu den Grundrechtsverletzungen des Bundesgerichtshofs wird Bezug genommen. Zusammenfassend ist für die Entscheidung des Dienstgerichtshofs festzustellen:

1. Die Entscheidung des Dienstgerichtshofs verstößt gegen Art. 97 Abs. 1 GG. Begriff und Bedeutung der richterlichen Unabhängigkeit werden verkannt. Es gibt keine zulässige Einschränkung oder Begrenzung der richterlichen Unabhängigkeit, wenn die Dienstaufsicht die Erzielung bestimmter Erledigungszahlen (Durchschnitt) anmahnt. Der Dienstgerichtshof hat im Übrigen - wie der Bundesgerichtshof - den Sachverhalt, aus dem sich eine (unzulässige) Einflussnahme durch den Bescheid vom 26.1.2012 auf die Rechtsanwendung des Beschwerdeführers ergibt, nicht subsumiert. Es wird ergänzend auf die entsprechenden Rügen in der Revisionsbegründung verwiesen (Anlage 27). 
2. Der Dienstgerichtshof hat das rechtliche Gehör des Beschwerdeführers (Art. 103 Abs. 1 GG) verletzt. Sachvortrag des Antragstellers wurde in großem Umfang nicht berücksichtigt, Beweisanträge wurden zu Unrecht abgelehnt. Es geht dabei, wie in der Entscheidung des Bundesgerichtshofs, um Umstände, die Inhalt und Bedeutung der im Ausgangsverfahren streitgegenständliche Maßnahme der Präsidentin charakterisieren (Einflussnahme auf die Rechtsanwendung des Beschwerdeführers). Der Beschwerdeführer hat die Rechtsverletzungen des Dienstgerichtshofs in der Revisionsbegründung erfolglos gerügt (vgl. die Anlage 27, S. 5 bis 8, S. 12 bis 15, S. 15 bis 21). Die Beweisbehauptungen des Beschwerdeführers I Ziff. 1, Ziff. 2 (Anlage 27, S. 11, 12) wurden trotz Wahrunterstellung vom Dienstgerichtshof bei der Entscheidungsfindung nicht berücksichtigt. Die Beweisanträge I. Ziff. 3, Ziff. 4, Ziff. 5, II. Ziff. 1, Ziff. 2, Ziff. 3 sowie IV. Ziff. 4, 5, 6. 7. wurden zu Unrecht abgelehnt. Wegen der Einzelheiten wird auf die Revisionsbegründung (Anlage 27, S. 15 bis 21) verwiesen.

3. Die Entscheidung des Dienstgerichtshofs ist willkürlich (Verletzung von Art. 3 Abs. 1 GG). Der Umgang mit dem Begriff der richterlichen Unabhängigkeit durch den Dienstgerichtshof ist rechtlich nicht vertretbar. Gesetzesbindung und Verpflichtung des Richters zur überzeugungsgemäßen Rechtsanwendung werden übergangen, obwohl diese Gesichtspunkte im Vordergrund des Berufungsverfahrens standen. Der Umgang des Dienstgerichtshofs mit großen Teilen des Sachverhalts, die sich auf die Bedeutung des Bescheids vom 26.1.2012 beziehen, ist schlechterdings nicht nachvollziehbar (vgl. die entsprechenden Ausführungen zur Verletzung von Art. 3 Abs. 1 GG durch den Bundesgerichtshof, oben VII. 1., 3. und 4.).

\section{Grundrechtsverstöße des Dienstgerichts für Richter}

Auch das Urteil des Dienstgerichts für Richter bei dem Landgericht Karlsruhe vom 4.12.2012 verletzt den Beschwerdeführer in seinen Grundrechten aus Art. 97 Abs. 1, Art. 103 Abs. 1 und Art. 3 Abs. 1 GG. Der Begriff der richterlichen Unabhängigkeit wird verkannt, Sachverhalt und tatsächliches Vorbringen des Beschwerdeführers wurden in großem Umfang nicht berücksichtigt, die Verkennung des Begriffs der richterlichen Unabhängigkeit und die Nichtberücksichtigung von wesentlichen Teilen des Sachverhalts sind unter keinem denkbaren Gesichtspunkt vertretbar und daher willkürlich. Es wird auf die entsprechenden Ausführungen zu den Grundrechtsverstößen des Dienstgerichtshofs verwiesen.

\section{Annahmevoraussetzungen}

1. Die Verfassungsbeschwerde ist zulässig und begründet (siehe oben).

2. Die Verfassungsbeschwerde ist zur Durchsetzung der in $\S 90$ Abs. 1 BVerfGG genannten Rechte angezeigt ( $\$ 93$ a Abs. 2 b) BVerfGG). 
Die Verfassungsbeschwerde ist notwendig, um die Rechte des Beschwerdeführers durchzusetzen. Nur mit der Verfassungsbeschwerde kann der Beschwerdeführer rechtlich sicherstellen, dass er in seiner Tätigkeit als Richter bis voraussichtlich 30.6.2022 weiterhin seiner Verpflichtung zur überzeugungsgemäßen Rechtsanwendung folgen kann. Der Präsident des Oberlandesgerichts Karlsruhe, Alexander Riedel, steht, wie sich aus seiner Prozessführung im dienstgerichtlichen Verfahren ergibt, bis heute auf dem Standpunkt, der Beschwerdeführer sei verpflichtet, dem Bescheid vom 26.1.2012 Folge zu leisten. Mit diesem Standpunkt ist die fortbestehende konkludente Androhung von Disziplinarmaßnahmen verbunden, für den Fall, dass sich der Beschwerdeführer dem Willen der Justizverwaltung in BadenWürttemberg nicht beugt.

Die Verfassungsbeschwerde ist zudem anzunehmen, um die grundrechtswidrige Praxis der Instanzgerichte im Ausgangsverfahren zu korrigieren. Vom Bundesverfassungsgericht ist eine grobe Verkennung der richterlichen Unabhängigkeit und der richterlichen Gesetzesbindung durch den Bundesgerichtshof und den Dienstgerichtshof richtig zu stellen (siehe im Einzelnen oben VII.). Die Annahme ist außerdem angezeigt, um erhebliche Unsicherheiten und Unklarheiten, die in der Rechtskultur an den Gerichten einerseits und in der Dienstaufsicht der Präsidentinnen und Präsidenten der Oberlandesgerichte andererseits entstanden sind, zu beseitigen (vgl. die Ausführungen zur Zulässigkeit der Verfassungsbeschwerde oben IV. 4. und die Anlagen 33 und 34).

3. Der Verfassungsbeschwerde kommt im Übrigen grundsätzliche verfassungsrechtliche Bedeutung zu (\$93 a Abs. 2 a) BVerfGG). Es geht um für die Praxis der Rechtsprechung wesentliche Fragen im Verhältnis zwischen der Exekutive und der Judikative.

\section{Politischer Hintergrund des Verfahrens}

\section{Erledigungsdruck in der ordentlichen Gerichtsbarkeit}

Der Konflikt zwischen dem Beschwerdeführer und der baden-württembergischen Justizverwaltung ist nur vor dem Hintergrund des in der deutschen Justiz herrschenden Erledigungsdrucks erklärbar, unter dem Richterinnen und Richter stehen. Dieser Hintergrund soll in Kurzform dargestellt werden.

a) Erledigungsdruck bedeutet, dass die Mehrheit der Richterinnen und Richter in Deutschland sich daran gewöhnt hat, dass alle ihnen an ihrem Gericht zugewiesen Verfahren von ihnen zu erledigen sind. Es ist zwar davon auszugehen, dass Richterinnen und Richter in der Regel sich weiterhin in ihrer Arbeit an das Gesetz halten wollen. Aber der Zeitbedarf pro Fall richtet sich in der Praxis in großem Umfang nach der Anzahl der zugewiesenen Fälle und weniger nach einer vom Erledigungsdruck unabhängig gebildeten richterlichen Überzeugung. Es findet bei der Rechts- 
anwendung eine Internalisierung des Erledigungsdrucks statt. Mit dem Begriff der „Sachgerechtigkeit", den der Bundesgerichtshof in der angegriffenen Entscheidung fern vom Boden des Grundgesetzes geprägt hat, wird diese Internalisierung des Erledigungsdrucks zum Maßstab der Rechtsanwendung erhoben.

b) Der Erledigungsdruck hat erhebliche Auswirkungen auf die Rechtsanwendung, im Zivilprozess insbesondere bei der Frage des rechtlichen Gehörs, im Umfang von Beweisaufnahmen, bei der Kenntnisnahme vom Akteninhalt, bei Vergleichsdruck auf die Parteien oder bei einer Suche nach dem ,dünnsten Brett“ für die Entscheidung. Am Oberlandesgericht lassen sich Auswirkungen besonders deutlich beobachten, weil die Rechtskultur in verschiedenen Senaten, bzw. unter verschiedenen Richtern, nach der Erfahrung des Beschwerdeführers schon immer vielfältig und unterschiedlich war. Dementsprechend ist der Spielraum, wie Richter am Oberlandesgericht auf den Erledigungsdruck reagieren können, besonders groß. Es ist kein Zufall, dass der Vertreter der Gegenseite in der mündlichen Verhandlung vor dem Dienstgericht in Karlsruhe am 4.12.2012 darauf hingewiesen hat, es gebe am Oberlandesgericht Karlsruhe Richter, die viermal so viele Fälle erledigen könnten wie der Beschwerdeführer.

c) Das Problem des Erledigungsdrucks hat sich in der Ziviljustiz in den letzten 25 Jahren deutlich verschärft. Ursache sind nicht gestiegene Eingangszahlen, sondern strukturelle Veränderungen der zur Entscheidung anstehenden Fälle. Sachverhalt und Rechtsfragen sind im Schnitt deutlich komplexer als in den zu bearbeitenden Fällen vor 25 Jahren. Ein Richter, der handwerklich genauso arbeiten möchte wie vor 25 Jahren, braucht heute im Schnitt pro Zivilrechtsfall die doppelte Zeit gegenüber den Verhältnissen vor 25 Jahren.

d) Der Erledigungsdruck führt aus psychologischen und soziologischen Gründen bei Richtern nahezu unausweichlich zu Konsequenzen in ihrem beruflichen Verhalten, die allerdings nur zum Teil reflektiert werden. Zwei Beispiele dokumentieren die Konsequenzen des Erledigungsdrucks in der Praxis:

- Im Rahmen der Deal-Entscheidung des Bundesverfassungsgerichts vom 19.3.2013 wurden Richter in Nordrhein-Westfalen nach ihrer Praxis befragt. Etwa die Hälfte der befragten Richter hat eingeräumt, dass sie gelegentlich oder regelmäßig Gesetzesvorschriften bewusst verletzen, um Strafverfahren zeitsparend zum Ende zu bringen.

- Thomas Fischer, inzwischen im Ruhestand, in seiner aktiven Zeit einer der selbstbewusstesten und intelligentesten Richter Deutschlands, hat in seinem Essay vom 9.6.2015 „Die Augen des Revisionsgerichts“ die Auswirkungen des Erledigungsdrucks für die Rechtskultur der Strafsenate am Bundesgerichtshof instruktiv dargestellt (vgl. den Text in der Anlage 36). Diese Kultur unterscheidet sich wegen des besonderen Erledigungsdrucks in Strafsachen sehr deutlich von der Entscheidungskultur der Zivilsenate am Bundesgerichtshof. 
e) $\mathrm{Zu}$ den Mechanismen des Erledigungsdrucks gehört, dass der Druck in großem Umfang mit dem Verhalten der Kollegen zusammenhängt. Das Zahlen- und Erledigungsdenken führt bei vielen Kollegen zu einer generellen Sorge, mehr Verfahren übernehmen zu müssen, wenn ein „Minderleister“" seine Verfahren nicht erledigt. Wer bei den Kollegen um Mitleid für seine begrenzten Fähigkeiten bittet, kann unter Umständen auch mit „schlechten Zahlen“ am Gericht weiter existieren. Wer, wie der Beschwerdeführer, pflichtgemäß eine Überlastungsanzeige an das Präsidium richtet, und dabei auf seine persönliche richterliche Überzeugung hinweist, die zu einem hohen Zeitbedarf führt, erntet in der Kollegenschaft hingegen Anfeindungen bis hin zum Hass.

f) Wegen des Erledigungsdrucks gibt es in der ordentlichen Gerichtsbarkeit keinen Zusammenhang zwischen niedrigen Erledigungszahlen einerseits und einer unterdurchschnittlichen Arbeitszeit von Richtern andererseits. Anderslautende Suggestionen, nicht nur von Vertretern der Justizverwaltungen, sondern nicht selten auch aus Teilen der Richterschaft, sind unzutreffend. Es gibt zwar wie in anderen Berufen, so auch bei Richtern, eine Minderheit, die weniger, gelegentlich auch deutlich weniger, als 40 Stunden pro Woche arbeitet. Jeder Richter, der weniger als 40 Stunden arbeitet, sorgt allerdings im Interesse seines psychischen und kollegialen Überlebens am Gericht durch seine Arbeitsweise dafür, dass die Erledigungszahlen „stimmen“, was insbesondere für einen Zivilrichter kein Problem ist, wenn er das will (s. o. b)).

g) Der Erledigungsdruck hat in den letzten 25 Jahren das Berufsethos in der Richterschaft negativ beeinflusst. Gute Erledigungszahlen sind nicht nur für Beförderungen, sondern auch für das Ansehen in der Kollegenschaft wichtiger als fachliche Aspekte der richterlichen Arbeit.

h) Es gibt in allen Bundesländern ein politisches Interesse der Landesregierungen und der Justizverwaltungen, den Erledigungsdruck in der Justiz aufrecht zu erhalten. Dieses Interesse ist aus der politischen Perspektive der Exekutive verständlich. Wenn keine Verfahren bei den Gerichten liegen bleiben, gibt es keine öffentliche Kritik am Justizminister; Kritik der Medien an der Sacharbeit der Richter, also an möglichen „Fehlurteilen“, kann sich nur gegen die verantwortlichen Richter richten. Wenn hingegen Verfahren teilweise unbearbeitet liegen bleiben, müssen sich die Justizverwaltungen und die Justizminister gegenüber der Öffentlichkeit rechtfertigen. Außerdem gibt es ein generelles haushaltspolitisches Interesse, dass sich die Richterinnen und Richter bei ihrer Arbeit vorrangig nach den unter politischen Aspekten bemessenen Ressourcen richten, und nicht etwa durch zeitaufwändige Arbeit „haushaltspolitische Vorgaben“ der Landesregierung und des Parlaments gefährden.

i) Das landespolitische Interesse an der Aufrechterhaltung des Erledigungsdrucks ist das entscheidende Motiv für die Maßnahme der Präsidentin des Oberlandesgerichts gegen den Beschwerdeführer. Die Präsidentin hat dies in ihrem Schriftsatz vom 29.4.2013 (Anlage 7) ausgeführt: „Durch die gesetzliche Vorgabe der Perso- 
nalausstattung und das tatsächliche Fallaufkommen wird aber der - auch für den Berufungsführer - verbindliche Maßstab aufgestellt, wie viel der einzelne Richter in seiner jeweiligen Funktion insgesamt zu erledigen hat." Das hochwirksame Prinzip des Erledigungsdrucks bedeutet aus politischer Perspektive, dass sich Richterinnen und Richter in Deutschland normalerweise von sich aus nach den haushaltspolitischen „Vorgaben der Personalausstattung“ richten. Da das psychologische Prinzip des Erledigungsdrucks im Fall des Beschwerdeführers nicht ausreichend wirkt, wurde aus der politischen Perspektive der Justizverwaltung die Maßnahme vom 26.1.2012 notwendig.

j) Die hohe Wirksamkeit des an den Gerichten herrschenden Binnendrucks ist im Übrigen der entscheidende Grund dafür, dass es in Deutschland kaum Richterinnen und Richter gibt, die versuchen, sich dem Druck in ähnlicher Weise aktiv zu widersetzen wie der Beschwerdeführer.

\section{Erfahrungen aus der Deal-Entscheidung des Bundesverfassungsgerichts vom 19.3.2013 - 2 BvR 2628/10 u. a. -}

In der bekannten Entscheidung vom 19.3.2013 hat sich der zweite Senat des Bundesverfassungsgerichts mit den Auswirkungen des Erledigungsdrucks in der Strafjustiz unter verfassungsrechtlichen Aspekten befasst. Das Ergebnis der Umfrage unter Richterinnen und Richtern in Nordrhein-Westfalen war bemerkenswert. Etwa die Hälfte der Richterinnen und Richter hat sich offen zu einer - gelegentlichen oder häufigen Rechtsanwendung bekannt, die von juristischen Kritikern teilweise als Rechtsbeugung bezeichnet wird. Die vom Gesetz nicht gedeckte Deal-Praxis der Richterinnen und Richter wurde vom Bundesverfassungsgericht zu Recht beanstandet.

Die Entscheidung des Bundesverfassungsgerichts vom 19.3.2013 erscheint in einem Teil der Begründung allerdings ergänzungsbedürftig. Wenn die Hälfte der Richter sich dazu bekennt, das Gesetz in bestimmten Fällen zu missachten, um Fälle effizient zu erledigen, dann reicht es nicht aus, allein an die Verantwortung der Richter - auch wenn diese nicht zu leugnen ist - zu appellieren. Vielmehr gibt es offensichtlich ein Strukturproblem, wenn derart viele Richter sich gezwungen fühlen, das Gesetz zu missachten. Für dieses Strukturproblem ist die Politik, also die jeweilige Landesregierung verantwortlich, welche die Justiz organisiert. Der zweite Senat hat in der Entscheidung vom 19.3.2013 nicht ausreichend zum Ausdruck gebracht, unter welchem enormen sozialen und psychischen Druck Richter stehen müssen, wenn sie sich derart weit von den Grundlagen ihres Berufes entfernen, wie die damals befragten Strafrichter in Nordrhein-Westfalen. Und der zweite Senat hat damals nicht erwähnt, welche auf der Hand liegenden politischen Interessen im Verhältnis zwischen der Exekutive in den Bundesländern einerseits und der Judikative andererseits das Strukturproblem des Erledigungsdrucks maßgeblich beeinflussen.

Im vorliegenden Verfahren erscheint es geboten, dass der Senat die Überlegungen zum Phänomen des Erledigungsdrucks in der Justiz aus der Entscheidung vom 19.3.2013 weiterführt: 
- Welches sind die politischen Interessen, die zu dem Konflikt zwischen dem Beschwerdeführer und der Justizverwaltung des Landes Baden-Württemberg geführt haben?

- Inwieweit entspricht es den rechtsstaatlichen Prinzipien des Grundgesetzes, wenn das Dienstgericht des Bundes die Mechanismen des Erledigungsdrucks an deutschen Gerichten legalisiert und zum Maßstab der Rechtsprechung in Deutschland erklärt? Darf die Justizverwaltung einen Richter in einem von vielen Richtern beobachteten Pilotverfahren zur Anpassung seiner Rechtsanwendung an die angeblichen „Vorgaben“ der Haushaltspolitik zwingen, wenn dieser Richter - anders als die meisten anderen Richterkollegen - sich nicht von sich aus dem herrschenden Erledigungsdruck durch Anpassung in seiner Rechtsanwendung beugt?

\section{Mögliche Folgewirkungen der Entscheidung über die Verfassungsbeschwerde}

- Es geht im Verhältnis zwischen Richtern einerseits und der Landesregierung anderseits um die Frage, wer möglicherweise - auch aus der Sicht der Öffentlichkeit und der Medien - die Verantwortung trägt, wenn es wegen einer zu langen Verfahrensdauer zu Unzuträglichkeiten an Gerichten kommt. Im Gespräch vom 18.10.2011 hat die Präsidentin des Oberlandesgerichts diesen Punkt selbst hervorgehoben. Sie müsse etwas unternehmen, da sie als Präsidentin nach dem Inkrafttreten des Gesetzes zum Rechtsschutz bei überlangen Gerichtsverfahren nicht für unbearbeitete Verfahren verantwortlich sein wolle.

- Die Ressourcen der Justiz, insbesondere die Anzahl der Richterstellen, bestimmt allein die Legislative im Haushaltsgesetz. Richter haben keine verfassungsrechtliche Kompetenz, die erforderlichen Ressourcen selbst zu bestimmen. Wenn Verfahren an einem Gericht trotz vollem Arbeitseinsatz der Richter nicht ausreichend gefördert werden, stellt sich allerdings die Frage nach möglichen Konsequenzen. Die Entscheidung über die Verfassungsbeschwerde kann Auswirkungen auf die Frage haben, inwieweit „Verfahrensrückstände“ an Gerichten eine Handlungsaufforderung für die Justizverwaltung (im Sinne eines Antrags an den Haushaltsgesetzgeber) und für den Haushaltsgesetzgeber selbst bedeuten können oder bedeuten müssen, und zwar unabhängig von fragwürdigen Bedarfsermittlungen durch das angeblich „objektive“" Personalbedarfsermittlungssystem „Pebbsy“.

- Die Mehrzahl der Präsidentinnen und Präsidenten der Oberlandesgerichte sieht bei der Dienstaufsicht über Richter, soweit es um „Zahlen“ und „Erledigungen“ geht, offenbar Zweckmäßigkeitsgesichtspunkte im Vordergrund und weniger die notwendigen rechtlichen und verfassungsrechtlichen Gesichtspunkte. So muss man wohl die Stellungnahme der Präsidentinnen und Präsidenten vom 12.7.2017 zur Umfrage der Neuen Richtervereinigung (Anlagen 33 und 34) verstehen. Vom Ergebnis der Verfassungsbeschwerde wird abhängen, in welchem Umfang Präsidentinnen und Präsidenten in der Zukunft politische Zweckmäßigkeitserwägungen zum Maßstab ihrer Dienstaufsicht machen können. 


\section{Begründung der Anträge}

1. Der mit der Verfassungsbeschwerde gestellte Antrag Ziff. 1 (siehe oben) folgt aus $\S 95$ Abs. 1, Abs. 2 1. Hs. BVerfGG.

2. Aus der Begründung der Verfassungsbeschwerde ergibt sich, dass im Falle einer Zurückverweisung einem Gericht des Ausgangsverfahrens kein Spielraum für eine Entscheidung bleiben würde. Es gibt auf der Grundlage des im Ausgangsverfahren unstreitigen Sachverhalts keinen Spielraum für unterschiedliche Auslegungen des Bescheids vom 26.1.2012. Der Bescheid ist daher wegen Verletzung der richterlichen Unabhängigkeit für unzulässig zu erklären. In einem derartigen Fall kann das Bundesverfassungsgericht durchentscheiden (vgl. Zuck, a.a.O., Rn. 1063 unter Hinweis auf die einschlägige Rechtsprechung des Bundesverfassungsgerichts).

Der Beschwerdeführer ist der Auffassung, dass das Bundesverfassungsgericht von dieser Möglichkeit des Durchentscheidens Gebrauch machen sollte. Das vom Beschwerdeführer angestrengte Verfahren läuft heute - zum Zeitpunkt dieser Verfassungsbeschwerde - bereits acht Jahre.

\section{Persönlicher Hintergrund des Verfahrens für den Beschwerde- führer}

Der Antragsteller ist am 25.6.1954 geboren, hat also bereits heute das 65. Lebensjahr vollendet. Das gerichtliche Verfahren, welches der Antragsteller gegen seinen Dienstherrn führt, dauert bereits acht Jahre. Zur Verfahrensdauer hat die Zurückverweisung im ersten Urteil des Bundesgerichtshofs vom 7.9.2017 beigetragen. Die Verfahrensverlängerung durch die Zurückverweisung war auch auf der Grundlage des damaligen eigenen Entscheidungskonzepts des Bundesgerichtshofs kaum nachvollziehbar. Dass sich das dienstgerichtliche Verfahren mit Vollendung des 65. Lebensjahres des Beschwerdeführers möglicherweise aus Rechtsgründen erledigen könnte, dürfte den Richterinnen und Richtern des Dienstgerichts des Bundes nicht verborgen geblieben sein.

Der Beschwerdeführer hat sich das dienstgerichtliche Verfahren nicht ausgesucht. Vor der Übergabe des Vermerks vom 12.10.2011 hätte der Beschwerdeführer es nicht für möglich gehalten, dass eine Präsidentin in Deutschland versuchen könnte, den vorhandenen Erledigungsdruck in der Justiz durch eine förmliche Maßnahme der Dienstaufsicht zu verschärfen, und eine „Vorgabe der Personalausstattung“ schriftlich zum Prinzip der Rechtsanwendung von Richtern in Deutschland zu erklären. Das Verfahren gegen den Dienstherrn ist am Ende des Arbeitslebens für den Beschwerdeführer zu einer nicht vorhergesehenen beruflichen Aufgabe geworden. Es gibt Menschen, die meinen, der Beschwerdeführer müsse das Verfahren durchstehen, um einem Abbau des Rechtsstaats in Deutschland entgegenzuwirken. Die Möglichkeit, das Verfahren durch die Verfassungsbeschwerde zum Abschluss zu bringen, war für den Beschwer- 
deführer einer der Gründe für seine Verlängerung der Dienstzeit, voraussichtlich bis zur Vollendung des 68. Lebensjahres im Juni 2022.

Der Beschwerdeführer stellt mit seinem Standpunkt das zahlen- und erledigungsorientierte Selbstverständnis vieler Richterinnen und Richter indirekt in Frage, ohne dass er selbst an dieser Konsequenz etwas ändern kann. Am Oberlandesgericht Karlsruhe hat sich daraus in der Kollegenschaft eine Stimmung gegen den Beschwerdeführer und seine Arbeit entwickelt, welche die sachliche Alltagsarbeit mit den Kolleginnen und Kollegen am Gericht außerordentlich erschwert. Die heutige Arbeitssituation hat sich für den Beschwerdeführer drastisch verschlechtert gegenüber der Situation vor Beginn der Maßnahmen der Präsidentin. Die Stimmung in der Kollegenschaft spiegelt sich u. a. in diskriminierenden Maßnahmen der Geschäftsverteilung, die es in dieser Form an keinem anderen Oberlandesgericht geben dürfte. Der Präsident des Oberlandesgerichts, Alexander Riedel, wurde in einem Interview im Deutschlandfunk vom 11.1.2019 zum Thema Mobbing am Oberlandesgericht Karlsruhe befragt. Die Antworten des Präsidenten lassen erkennen, dass er die gegen den Beschwerdeführer gerichtete Stimmung am Oberlandesgericht unterstützt (vgl. den Text des Interviews in der Anlage 37).

\section{Veröffentlichungen zum Verfahrensgegenstand}

Es hat zum Verfahrensgegenstand verschiedene rechtliche Stellungnahmen gegeben. Es ist hinzuweisen auf die Aufsätze von Fabian Wittreck, ,Richterliche Erledigungszahlen als Gegenstand der Dienstaufsicht“ (NJW 2012, 3287, Anlage 35) und „Erledigungszahlen unter (Dienst-)Aufsicht“ (DRiZ 2013, 60, Anlage 38), auf den Beitrag „Die Richtgeschwindigkeit der Justiz“ (Frankfurter Allgemeine - Einspruch Magazin vom 29.11.2017, Anlage 39) von Carsten Schütz und derselbe „Richterliche Erledigungsfixierung verändert das Recht“ in NJW-aktuell 40/2016 (Anlage 40). Der Beschwerdeführer weist außerdem auf ein Streitgespräch hin, welches er am 9.5.2020 mit dem Präsidenten des Oberlandesgerichts Frankfurt, Roman Poseck, geführt hat (Frankfurter Allgemeine - Einspruch Magazin, Anlage 41).

\section{Anlagenverzeichnis:}

1. Vollmacht für das Verfahren der Verfassungsbeschwerde

2. Urteil des Bundesgerichtshofs vom 12.5.2020

3. Urteil des Dienstgerichtshofs für Richter bei dem Oberlandesgericht Stuttgart vom 21.5.2019

4. Urteil des Dienstgerichts für Richter bei dem Landgericht Karlsruhe vom 4.12.2012 
5. Bescheid der Präsidentin des Oberlandesgerichts Karlsruhe vom 26.1.2012

6. Widerspruchsbescheid vom 20.4.2012

7. Schriftsatz der Präsidentin des Oberlandesgerichts vor dem Dienstgerichtshof vom 29.4.2013

8. Vermerk des Beschwerdeführers vom 6.11.2011 (Gespräch vom 30.4.2010)

9. Vermerk der Präsidentin des Oberlandesgerichts Karlsruhe vom 12.10.2011

10. Schreiben des Präsidenten des Oberlandesgerichts an die Presse vom 2.2.2017

11. Berufungsbegründung zum Dienstgerichtshof vom 11.3.2013

12. Grundsatzschriftsatz zum Dienstgerichtshof vom 27.11.2013

13. Schriftsatz zum Dienstgerichtshof vom 23.1.2015

14. Protokoll der mündlichen Verhandlung des Dienstgerichtshofs vom 17.4.2015

15. Urteil des Dienstgerichtshofs vom 17.4.2015

16. Revisionsbegründung zum Bundesgerichtshof vom 23.9.2015

17. Revisionserwiderung des Präsidenten des Oberlandesgerichts Karlsruhe vom 26.1.2016

18. Plädoyer des Beschwerdeführers im Termin vom 7.9.2017 beim Bundesgerichtshof

19. Urteil des Bundesgerichtshofs vom 7.9.2017

20. Anhörungsrüge zum Bundesgerichtshof vom 13.11.2017

21. Beschluss des Bundesgerichtshofs vom 13.12.2017

22. Zustellungsurkunde vom 2.1.2018

23. Verfassungsbeschwerde - 2 BvR 174/18 - vom 30.1.2018

24. Beschluss des Bundesverfassungsgerichts - 2 BvR 174/18 - vom 9.3.2018

25. Schriftsatz zum Dienstgerichtshof vom 16.4.2018

26. Schriftsatz zum Dienstgerichtshof vom 9.5.2019 
27. Revisionsbegründung zum Bundesgerichtshof vom 7.10.2019

28. Plädoyer des Beschwerdeführers vor dem Bundesgerichtshof vom 12.5.2020

29. Anhörungsrüge zum Bundesgerichtshof vom 10.6.2020

30. Beschluss des Bundesgerichtshofs vom 7.7.2020

31. Zustellungsurkunde vom 20.7.2020

32. Urteil des Dienstgerichts für Richter bei dem Landgericht Karlsruhe im Parallelverfahren RDG 5/12vom 4.12.2012 (betreffend den Vermerk vom 12.10.2011)

33. Umfrage der Neuen Richtervereinigung an die Präsidentinnen und Präsidenten der Oberlandesgerichte vom 16.6.2017

34. Antwortschreiben der Präsidentinnen und Präsidenten vom 12.7.2017

35. Aufsatz von Fabian Wittreck, NJW 2012, 3287

36. Thomas Fischer, Die Augen des Revisionsgerichts, 9.6.2015

37. Interview des Präsidenten des Oberlandesgerichts Karlsruhe, Alexander Riedel, im Deutschlandfunk vom 11.1.2019

38. Aufsatz von Fabian Wittreck, DRiZ 2013, 60

39. Aufsatz von Carsten Schütz, Frankfurter Allgemeine - Einspruch Magazin vom 29.11.2017

40. Beitrag von Carsten Schütz, NJW-aktuell 40/2016

41. Streitgespräch des Beschwerdeführers mit dem Präsidenten des Oberlandesgerichts Frankfurt, Roman Poseck, vom 9.5.2020 